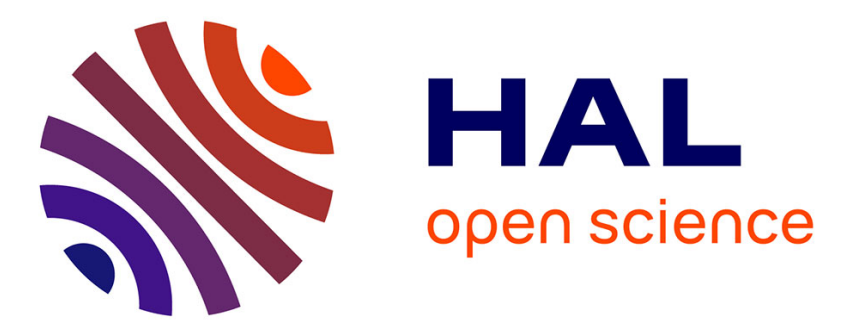

\title{
Connecting the Properties of Coronal Shock Waves with Those of Solar Energetic Particles
}

Athanasios Kouloumvakos, Alexis Rouillard, Yihong Wu, Rami Vainio, Angelos Vourlidas, Illya Plotnikov Plotnikov, Alexandr Afanasiev, Hakan Önel

\section{- To cite this version:}

Athanasios Kouloumvakos, Alexis Rouillard, Yihong Wu, Rami Vainio, Angelos Vourlidas, et al.. Connecting the Properties of Coronal Shock Waves with Those of Solar Energetic Particles. The Astrophysical Journal, 2019, 876 (1), pp.80. 10.3847/1538-4357/ab15d7 . hal-02415623

\section{HAL Id: hal-02415623 \\ https://hal.science/hal-02415623}

Submitted on 17 Dec 2019

HAL is a multi-disciplinary open access archive for the deposit and dissemination of scientific research documents, whether they are published or not. The documents may come from teaching and research institutions in France or abroad, or from public or private research centers.
L'archive ouverte pluridisciplinaire HAL, est destinée au dépôt et à la diffusion de documents scientifiques de niveau recherche, publiés ou non, émanant des établissements d'enseignement et de recherche français ou étrangers, des laboratoires publics ou privés. 


\title{
Connecting the Properties of Coronal Shock Waves with Those of Solar Energetic Particles
}

\author{
Athanasios Kouloumvakos ${ }^{1}$, Alexis P. Rouillard ${ }^{1}$, Yihong $\mathrm{Wu}^{1}$, Rami Vainio ${ }^{2}$, Angelos Vourlidas ${ }^{3}$ (i), Illya Plotnikov ${ }^{1,4}$, \\ Alexandr Afanasiev ${ }^{2}$, and Hakan Önel ${ }^{5}$ \\ ${ }^{1}$ IRAP, Université Toulouse III-Paul Sabatier, CNRS, CNES, Toulouse, France; akouloumvako@irap.omp.eu \\ ${ }^{2}$ University of Turku, Finland \\ ${ }^{3}$ John Hopkins APL, Laurel, MD 20723, USA \\ ${ }^{4}$ Department of Astrophysical Sciences, Princeton University, 4 Ivy Lane, Princeton, NJ 08544, USA \\ ${ }^{5}$ Leibniz Institute for Astrophysics Potsdam, Babelsberg, Germany \\ Received 2019 February 21; revised 2019 March 31; accepted 2019 April 2; published 2019 May 7
}

\begin{abstract}
We develop and exploit a new catalog of coronal pressure waves modeled in 3D to study the potential role of these waves in accelerating solar energetic particles (SEPs) measured in situ. Our sample comprises modeled shocks and SEP events detected during solar cycle 24 observed over a broad range of longitudes. From the 3D reconstruction of shock waves using coronagraphic observations we derived the 3D velocity along the entire front as a function of time. Combining new reconstruction techniques with global models of the solar corona, we derive the 3D distribution of basic shock parameters such as Mach numbers, compression ratios, and shock geometry. We then model in a time-dependent manner how the shock wave connects magnetically with spacecraft making in situ measurements of SEPs. This allows us to compare modeled shock parameters deduced at the magnetically wellconnected regions, with different key parameters of SEPs such as their maximum intensity. This approach accounts for projection effects associated with remote-sensing observations and constitutes the most extensive study to date of shock waves in the corona and their relation to SEPs. We find a high correlation between the maximum flux of SEPs and the strength of coronal shock waves quantified, for instance, by the Mach number. We discuss the implications of that work for understanding particle acceleration in the corona.
\end{abstract}

Key words: shock waves - Sun: coronal mass ejections (CMEs) - Sun: particle emission

\section{Introduction}

The origin of solar energetic particles (SEPs) measured in situ in the inner heliosphere at energies ranging from a few hundred $\mathrm{keV}$ up to $10 \mathrm{GeV}$ is still debated. Solar flares and shock waves that develop in the solar corona during coronal mass ejections (CMEs) are two known particle accelerators. The charged particles propagate from their production site through the interplanetary medium, gyrating along the magnetic field lines until their detection in situ. The difficulty identifying which accelerator contributes to the acceleration process resides in the great distances that separate the location where energetic particles are produced in the corona and their point of detection in situ, typically situated near 1 au. The Parker Solar Probe (PSP) launched recently will enter the solar corona and for the first time will get closer to the accelerator and should deliver over the next years new critical information on the acceleration mechanisms of energetic particles. However, $P S P$ is an encounter mission and will not provide a continuous and systematic coverage of solar eruptions, and complementary observations from other missions such as the Solar-Terrestrial Relation Observatory (STEREO) will be necessary to gain a more complete picture of the coronal dynamics involved in the energization process of particles.

STEREO has provided over $10 \mathrm{yr}$ of continuous monitoring of CMEs from the Sun to Earth-like distances, as well as multipoint measurements of SEPs in the $\mathrm{keV}$ to $100 \mathrm{MeV}$ energy range. These observations have revealed a number of puzzling new properties of SEPs. For instance, gradual and impulsive SEP events have been measured over extended ranges of longitudes by STEREO, sometimes extending over $360^{\circ}$ around the Sun (Dresing et al. 2012; Rouillard et al. 2012;
Lario et al. 2014). It is yet unclear how flares or even coronal shocks could produce SEPs over such extended regions of the inner heliosphere, and various studies have searched for the possible role of particle transport in the form of cross-field diffusion (e.g., Giacalone et al. 2000; Zhang et al. 2003).

Diffusive shock acceleration (DSA) is one of several mechanisms proposed to explain the energization process of particles at CME-driven shocks. In DSA, the properties of the energetic particles that are locally accelerated by a propagating shock wave are expected to be closely related to (1) the critical properties of the shock, such as the Mach numbers, compression ratios, and the shock geometry (e.g., simulations; Kozarev \& Schwadron 2016; Afanasiev et al. 2018); (2) the seed particle population (Kahler 2001; Desai et al. 2006; Tylka \& Lee 2006; Sandroos \& Vainio 2007; Vainio et al. 2017); and (3) the acceleration timescale, which is related to the level of particle scattering off magnetic irregularities. The properties of shock waves are related to the expansion speed of CMEs driving the shock and the background medium through which the shock forms, both of which exhibit significant event-to-event variability. In addition, for a single CME, the properties of shock waves are likely to vary significantly across the shock surface (e.g., Kouloumvakos et al. 2014; Rouillard et al. 2016; Kwon \& Vourlidas 2017, 2018). It is therefore likely that the great variability of gradual SEP events stems to a large extent from event-specific conditions and how a particular probe making in situ measurements connects to the shock. To verify these theoretical predictions, we need to connect shock properties with in situ measurements by accounting for the properties of each specific event. 
Shock properties are difficult to infer from remote-sensing observations. Previous statistical studies of SEP events have focused on comparisons between CME and SEP properties. A correlation of SEP peak intensities with the speed and other properties of the associated CMEs has been derived in several studies (e.g., Kahler et al. 1999; Reames 1999; Kahler 2001; Kahler \& Vourlidas 2005, 2013; Richardson et al. 2015; Papaioannou et al. 2016). Kahler (2001) showed that there is a correlation between proton peak intensities and the speed of the associated CMEs. Kahler \& Vourlidas (2013) found a good correlation between the total CME energy and the SEP peak intensity or the SEP energy integrated over the whole event. Richardson et al. (2015) compared the estimates of CME parameters using several catalogs. Most CME studies based on LASCO images alone suffer from projection effects associated with the optically thin corona. There have been different attempts to remove these ambiguities using strategic quadrature configurations of the inner heliospheric probes such as STEREO. These studies confirm a correlation between SEP peak intensity and CME speed.

These studies reveal a certain trend, but they are by no means tight correlations, as significant spread is seen on scatter plots of SEP fluxes versus CME speed. This scatter could be related to an inadequate choice and estimate of shock properties because they are projected measures in the plane of the sky (e.g., Colaninno \& Vourlidas 2009), the fact that more subtle effects come into play such as seed particle populations (e.g., Kahler 2001; Cane et al. 2003; Tylka et al. 2005; Sandroos \& Vainio 2007; Vainio et al. 2017; Reames 2018), shock geometry (Sandroos \& Vainio 2009), or the fact that the shock is not the prime accelerator of these particles.

Although there are difficulties in connecting shock properties with SEP events, significant advances have been achieved in recent years to determine $3 \mathrm{D}$ shock parameters from coronal imagery. The techniques now provide estimates of density compression ratio from remote-sensing observations (Ontiveros \& Vourlidas 2009; Bemporad \& Mancuso 2011; Kouloumvakos et al. 2014; Kwon \& Vourlidas 2018). A combination of shock forward modeling with MHD simulations of the background corona (Rouillard et al. 2016) can provide a complete mapping of shock properties that are likely relevant to the energization of particles. Recent studies based on this approach have exploited STEREO imagery for half a dozen events and have revealed a connection between the longitudinal spread (i.e., $>90^{\circ}-180^{\circ}$ ) and timing (i.e., $>2 \mathrm{hr}$ ) of SEP events and the spatial and temporal evolution of coronal and interplanetary shock waves driven by fast CMEs (Rouillard et al. 2012, 2016; Lario et al. 2014, 2016, 2017; Kouloumvakos et al. 2016).

More specifically, Reames (2012) compared critical shock parameters and SEP characteristics at 1 au and suggested a link between the strength of the shock and the SEPs measured in situ. He investigated the connection of various interplanetary shock parameters with the properties of accelerated particles measured in situ and found that IP shocks with high shock speed, high shock compression ratio, and highly oblique shocknormal angles $\left(\theta_{\mathrm{Bn}}\right)$ lead to the most efficient particle acceleration. The detailed study by Rouillard et al. (2016) of a single event seems to validate this expectation.

The aim of this paper is to examine consistently the comparison between critical coronal shock wave parameters derived from 3D modeling and observations and SEP characteristics. We improve past studies that connect the shocks/CMEs to SEP properties by alleviating projection effects, accounting for both the complexities of coronal shocks and how they are likely to connect magnetically with in situ spacecraft. We improve further the Rouillard et al. (2016) methods and perform an extensive study of the 3D shock modeled parameters in connection with the SEP characteristics. We first present in Section 2 the different data sets and techniques employed for the SEPs and the derivation of 3D shock properties. We then present in Section 3 the statistical relationship between the shock parameters and the SEP characteristics. Finally, in Section 4 we summarize and discuss our results.

\section{Data Analysis}

\subsection{Selection of SEP Events}

For our analysis we use the SEP event list given in Table 3 of Paassilta et al. (2018), which consists of 46 SEP events with energies greater than $50 \mathrm{MeV}$ that occurred from 2009 to 2016. This catalog is based on measurements of energetic protons by the Energetic and Relativistic Nuclei and Electron experiment (ERNE; Torsti et al. 1995) on board the Solar and Heliospheric Observatory (SOHO) and the High Energy Telescope (HET; von Rosenvinge et al. 2008) on board STEREO. An SEP event was selected when particle fluxes increased by a factor of $\geqslant 3.0$ over the quiet-time background fluxes of at least two of the three spacecraft (i.e., SOHO, STEREO-A, or STEREO-B) over a short time interval. We added to this SEP list the 2017 September 10 event detected by STA, SOHO/ERNE, and the Geostationary Operational Environmental Satellites (GOES).

From the initial catalog of the 47 SEP events we rejected 14 events ${ }^{6}$ because of insufficient or bad-quality data (in situ or remote sensing). In 8 of the 14 events the energetic protons were detected in only two of the three spacecraft. Insufficient or missing data were retrieved at one or both instruments near the onset and/or the rise phase of the SEP events. This made it impossible to accurately determine the SEP characteristics; therefore, we do not perform any further analysis for those events. In two of the remaining six events there was insufficient coronal imaging to perform a reliable $3 \mathrm{D}$ fitting of the coronal pressure waves (see Section 2.3.1). Additionally, in three events multiple CMEs occurred in quick succession, making it difficult to determine the evolution of the shock wave and consequently to constrain accurately the shock fittings. The final list with the remaining 33 SEP events is given in Table 1.

The final catalog includes 12 SEP events with clear detection of energetic protons at the three different locations (e.g., ERNE in L1 or GOES, STA, and STB) and 21 events for which the energetic protons were detected clearly in at least two locations. It is worth noting that for those 21 events we have 12 events for which high-energy protons were clearly not detected at the third available location, despite the availability and good quality of the particle data, and 6 events with ambiguous detection, i.e., low intensities and delayed onsets at the third location. For the remaining 3 cases there was a data gap in the particle observations during SEP events at the third location. In events with data gaps on $S O H O / E R N E$ we use the GOES data

\footnotetext{
6 The SEP events of 2011 January 28, 2012 July 8 and 12, 2012 November 8, 2013 April 11 and 24, 2013 May 15, 2013 August 20, 2013 September 30, 2013 December 26, 2014 March 4, 2014 September 25, 2014 December 13 and 2015 July 1.
} 
Table 1

List of the SEP Events Analyzed in This Study

\begin{tabular}{|c|c|c|c|}
\hline $\begin{array}{l}\text { Event Day } \\
\text { (1) }\end{array}$ & $\begin{array}{c}\text { Flare SXR } \\
\text { (2) }\end{array}$ & $\begin{array}{l}\text { Location } \\
\text { (3) }\end{array}$ & $\begin{array}{c}\text { CME Speed } \\
\text { (4) }\end{array}$ \\
\hline & Class (Onset) & Lat. Lon. & $\left(\mathrm{km} \mathrm{s}^{-1}\right)$ \\
\hline 2011 Feb 15 & X2.2 (01:44) & S20 W12 & 1161 \\
\hline 2011 Mar 7 & M3.7 (19:43) & N30 W48 & 2125 \\
\hline 2011 Mar 21 & $\cdots$ & N16 W130 & 1341 \\
\hline 2011 Aug 4 & M9.3 (03:41) & N19 W36 & 1761 \\
\hline 2011 Sep 6 & X2.1 (22:12) & N14 W18 & 728 \\
\hline 2011 Sep 22 & X1.4 (10:29) & N09 E89 & 1905 \\
\hline 2011 Oct 4 & $\ldots$ & N33 E151 & 1101 \\
\hline 2011 Nov 3 & $\ldots$ & N12 E152 & 1017 \\
\hline 2012 Jan 23 & M8.7 (03:38) & N18 W25 & 2175 \\
\hline 2012 Jan 27 & X1.7 (17:37) & N27 W71 & 2508 \\
\hline 2012 Mar 5 & X1.1 (02:30) & N16 E54 & 1531 \\
\hline 2012 Mar 7 & X5.4 (00:02) & N17 E27 & 2684 \\
\hline 2012 Mar 24 & $\cdots$ & N11 E166 & 1451 \\
\hline 2012 May 17 & M5.1 (01:25) & N11 W76 & 1582 \\
\hline 2012 Jul 23 & $\cdots$ & S23 W137 & 2003 \\
\hline 2012 Sep 20 & $\cdots$ & S11 E159 & 2458 \\
\hline 2012 Sep 28 & C3.7 (23:36*) & N09 W32 & 1135 \\
\hline 2013 Mar 5 & $\ldots$ & N10 E145 & 1316 \\
\hline 2013 May 22 & M5.0 (13:08) & S18 W15 & 2126 \\
\hline 2013 Oct 5 & $\cdots$ & S24 E121 & 964 \\
\hline 2013 Oct 11 & M1.5 (07:01) & S21 E44 & 1200 \\
\hline 2013 Oct 25 & X1.7 (07:53) & S08 E73 & 587 \\
\hline 2013 Oct 28 & M4.4 (15:07) & S08 E28 & 812 \\
\hline 2013 Nov 2 & $\cdots$ & N06 W133 & 963 \\
\hline 2013 Nov 7 & $\cdots$ & N02 E151 & 1405 \\
\hline 2013 Dec 28 & $\cdots$ & S13 W125 & 1118 \\
\hline 2014 Jan 6 & C2.1 (07:27) & S15 W89 & 1402 \\
\hline 2014 Jan 7 & X1.2 (18:04) & S12 W08 & 2441 \\
\hline 2014 Feb 25 & X4.9 (00:39) & S12 E82 & 2147 \\
\hline 2014 Mar 5 & $\cdots$ & N12 E179 & 1045 \\
\hline 2014 Sep 1 & $\cdots$ & N14 E128 & 1901 \\
\hline 2014 Sep 10 & X1.6 (17:21) & N14 E2 & 1267 \\
\hline 2017 Sep 10 & X8.2 (15:35) & S08 W88 & 3163 \\
\hline
\end{tabular}

to complement the observations; this procedure is explained in Section 2.2. For the above separation we have already considered the GOES data. In summary, we have $84 \mathrm{~S} / \mathrm{C}$ specific SEP events (including the six ambiguous spacecraft (S/C)-specific cases).

A simple consideration of the source locations of the $84 \mathrm{~S} / \mathrm{C}$ SEP events in our list by accounting for the Parker spiral for a wind speed measured near the onset of the SEPs reveals that 30 events measured at specific spacecraft were connecting magnetically to within $\Delta \Phi_{c}=50^{\circ}$ of the flaring region, e.g., cases with presumably close magnetic connections and 54 cases with more distant magnetic connections $\left(\Delta \Phi_{c}>50^{\circ}\right)$. Past observations have revealed that the onset of SEP events is prompt when magnetic connectivity is established with the nose of interplanetary shocks (i.e., along the central axis of the CME driver), but the rise in SEP fluxes is more gradual when magnetic field lines connect to regions far from the shock nose (Cane et al. 1988; Reames 1999; Cane \& Lario 2006; Reames 2013). This trend has been confirmed from detailed reconstructions of shock waves, at least 30 minutes into the event, when the shock occupies already a significant portion of the solar disk (see Rouillard et al. 2012, 2016; Lario et al. 2014, 2016, 2017; Kouloumvakos et al. 2016; Patsourakos et al. 2016). It remains to be determined whether the flanks of the shock could be significant particle accelerators during the very early expansion phase of CMEs within 10-20 minutes of the CME onset.

In 20 SEP events the source region was located on the visible disk as viewed from Earth and a flare detection was possible by the GOES spacecraft. For those 20 events, 11 are associated with an X-class, 7 with an M-class, and 2 with a C-class flare measured in soft X-rays by GOES. In 13 SEP events the eruption was back-sided. Additionally, from an inspection of the available CME properties from the CDAW SOHO/LASCO $\mathrm{CME} \mathrm{Catalog}^{7}$ and the Dual-Viewpoint CME Catalog from the STEREO coronagraphs ${ }^{8}$ (Vourlidas et al. 2017) we found that our SEP events are associated with wide halo CMEs, with projected speeds that range from 580 to $3163 \mathrm{~km} \mathrm{~s}^{-1}$, with a mean value of $\sim 1519 \mathrm{~km} \mathrm{~s}^{-1}$.

Additionally, from an inspection of the available radio spectral quick-look data from the SECCHI-radio-survey ${ }^{9}$ and the data from the RSTN radiospectrographs, we found that type II radio bursts (in metric, decametric, or kilometric wavelengths) occurred in all SEP events of our list. Furthermore, in 29 events the type II bursts have an interplanetary counterpart (i.e., observed by WIND/WAVES or SWAVES). For those 29 cases, in $\sim 65 \%$ (17/29 events) the IP-type II emission persists for longer than 3-4 hr. In some extreme events the type II emission can be clearly tracked over half a day; a clear example is the "Carrington-like" event of 2012 July 23. The presence of a type II radio burst in all SEP events in our list implies that shocks formed during all the events.

\subsection{Calculation of SEP Event Peak Intensities}

In our analysis we focus on the relationship between the shock properties and the SEP flux peak intensities. We use the 1-minute-averaged data of energetic proton fluxes from SOHO/ERNE and STEREO/HET instruments. SOHO/ERNE performs measurements of energetic protons in 20 individual energy channels spanning from 1.58 to $131 \mathrm{MeV}$. Its two separate detectors ERNE/LED and ERNE/HED provide 10 individual channels each. In our study we use data only from the HED detector in energies ranging from 13.8 to $131 \mathrm{MeV}$ (i.e., channels 11-20). STEREO/HET performs measurements in 11 individual energy channels ranging from 13.6 to $100 \mathrm{MeV}$. Additionally, we use 5-minute-averaged particle data from $G O E S^{10}$ to perform a correction of the $S O H O / E R N E$ observations from saturation due to its large geometric factor. In our analysis we focus on three energy ranges, 20-26 MeV, 40-60 MeV, and 60-100 MeV. Those were chosen to roughly match the individual energy channels of the different instruments we use. Also those ranges are frequently employed in previous statistical studies of SEP characteristics (Kahler 2001; Richardson et al. 2014; Papaioannou et al. 2016).

Since the division of the proton energy channels is not identical between ERNE and HET instruments, we combine adjacent channels to obtain matching energy ranges. For the energy range of 20-26 MeV, we use the channels ch-4 and ch-5

\footnotetext{
7 Available at https://cdaw.gsfc.nasa.gov/CME_list/.

8 Available at http://solar.jhuapl.edu/Data-Products/COR-CME-Catalog.php.

9 http://secchirh.obspm.fr/. This survey is generated and maintained the Observatoire de Paris by the LESIA UMR CNRS 8109 in cooperation with the Artemis team, Universities of Athens and Ioannina, and the Naval Research Laboratory.

${ }^{10}$ We used the calibrated GOES data that are provided from the Solar Energetic Particle Environment Modelling (SEPEM) application server.
} 

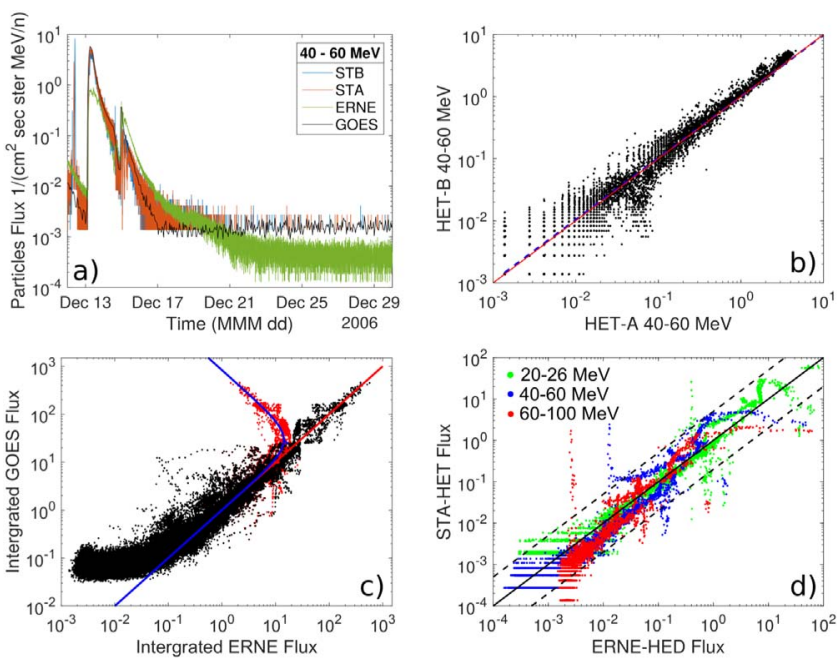

Figure 1. (a) Observations and (b)-(d) comparisons of the STEREO/HET, $S O H O / E R N E$, and GOES energetic proton intensities. Panel (a) shows the observations of proton intensities during 2006 December, in the 40-60 MeV energy range. Panel (b) shows the comparison of STEREO/HET's proton intensities in the same time interval. Panel (c) shows the comparison between SOHO/ERNE and GOES-SEPEM in an extended time interval from 2010 to 2014, before (red points) and after (blue points) ERNE's saturation correction. Panel (d) shows the comparison, during 2006 December, between STEREO-A/ HET and $\mathrm{SOHO} / \mathrm{ERNE}$ after their intercalibration. Saturation corrections has been applied to ERNE, and the data have been resampled to 5-minute-averaged values. The solid line depicts the one-to-one relation, and the dashed lines show the $5 \times$ difference.

of STEREO/HET with energies spanning from 20.8 to $23.8 \mathrm{MeV}$ (geometric mean: $22.2 \mathrm{MeV}$ ) and from 23.8 to 26.4 MeV (geometric mean: $25.1 \mathrm{MeV}$ ), respectively. The resulting mean energy ${ }^{11}$ for the HET composite channel is $23.4 \mathrm{MeV}$, which closely matches the mean energy of $\mathrm{SOHO} /$ ERNE ch-13 (23.3 MeV). At the energy ranges $40-60 \mathrm{MeV}$ and $60-100 \mathrm{MeV}$, we have considered ch-10 and ch-11, respectively, of STEREO/HET (mean energy: 49.0 and $77.5 \mathrm{MeV}$, respectively). Those channels have exactly the same energy range as the two pre-selected intervals of 40-60 $\mathrm{MeV}$ and 60-100 MeV. We combine the ERNE ch-16 with ch17 and ch-18 with ch-19 to produce two individual channels in the energy ranges of 40.5-67.3 $\mathrm{MeV}$ and 63.8-101 MeV. The resulting mean energies for the two composite ERNE channels are 52.0 and $80.0 \mathrm{MeV}$, respectively, and reasonably match the energies of the corresponding HET channels we use.

We perform an intercalibration of our particle data to ensure that the measured proton intensities are comparable among the various instruments in the selected energy intervals. We follow the method in Richardson et al. (2014), and we compare the proton fluxes in the three energy ranges of $20-26 \mathrm{MeV}$, 40-60 MeV, and 60-100 MeV during 2006 December. At that time the two STEREO spacecraft were close to Earth; therefore, the in situ measurements between STEREO and the instruments in L1 should be comparable. To perform the calibration, we start the calibration with the SEP event that occurs at 2006 December 13, and we continue until the end of the month, where the intensities were close to the background values. In this way we have a large dynamic range of energetic particle intensities to perform the correlations. In Figure 1 we show a

\footnotetext{
11 We will use the term "mean energy" of an energy channel to describe its geometric mean value.
}

part of the results from the intercalibration. In panel (a) we show the energetic proton measurements from STEREO/HET and $S O H O / E R N E$ in $40-60 \mathrm{MeV}$ during the calibration interval (e.g., from 2006 December 13 to 31).

In Figure 1(b) we compare the energetic proton fluxes between STEREO/HET-A and HET-B. From the comparison of the particle data between HET-A and HET-B we find that the particle intensities at both STEREOs are highly correlated and comparable in every energy range considered here, during the selected time interval. This result is expected and is in accordance with the results presented by Richardson et al. (2014). The residual differences in the particle fluxes may be due to anisotropies in the particle distribution and the different pointing directions of the individual particle telescopes (see von Rosenvinge et al. 2009). In 2006 December STEREO-B was inverted so that HET-B was observing perpendicular to the nominal Parker spiral direction whereas HET-A was observing along the spiral direction. Therefore, in our analysis we use the energetic proton data from STEREO-A and STEREO-B without applying any correction. We note that these particle intensity relations have a mean absolute percentage error of $\sim 12 \%-27 \%$, depending on the energy channel. This sets a lower limit to the uncertainty in the determined peak proton intensities.

From the comparison of the particle data between STEREO/ HET and SOHO/ERNE we find a rather complicated relation. In every energy range we observe (1) a break in the linear relationship between HET-A and ERNE at medium to high intensities (see Figure 3 of Richardson et al. 2014) and (2) a turnover at high intensities where $\mathrm{SOHO} / \mathrm{ERNE}$ saturates. Before we proceed with the cross-calibration, we perform a correction of the ERNE saturation effect using GOES-SEPEM data from 2010 to 2014. The comparison of the particle data between GOES and ERNE is shown in Figure 1(c), before (red points) and after (black points) the saturation correction. The intensities we show in this panel are integral intensities over all ERNE and GOES energies $(\sim 13-130 \mathrm{MeV})$. The ERNE response saturates like a paralyzable instrument: once the true flux exceeds a certain value, the measured one starts to decrease. The appropriate methodology is used to correct ERNE's intensities from saturation (see Wurz et al. 2007).

Before the final calibration between the HET and ERNE, we resample the data to 5-minute-averaged values. This averaging smooths out any time differences of the measurements at different positions. In Figure 1(c) we show the comparison of the particle data between the STA-HET and SOHO/ERNE data after the saturation correction and the calibration. After the calibration, the proton intensity relations result in a mean absolute percentage error of $\sim 30 \%-80 \%$, depending on the energy channel. The final scaling functions for the intercalibrated ERNE intensities with STEREO/HET have the form $I^{\prime}=a I$. For the $20-26 \mathrm{MeV}$ energy channel we have $a=2.15$, for $40-60 \mathrm{MeV}, a=0.73$, and for $60-100 \mathrm{MeV}$, $a=2.55$. In our statistical analysis we use the corrected/ calibrated ERNE observations, and only in cases where there are gaps in the observations do we use the GOES-SEPEM data instead.

From the intercalibrated particle data we determine the peak intensities (Ip) in the three selected energy ranges. For the purpose of this study we are limited to measuring the Ip when the associated shocks are still close to the Sun and exclude the peaks associated with the arrival of interplanetary shocks at the instruments, when detected in situ. To identify the possible 


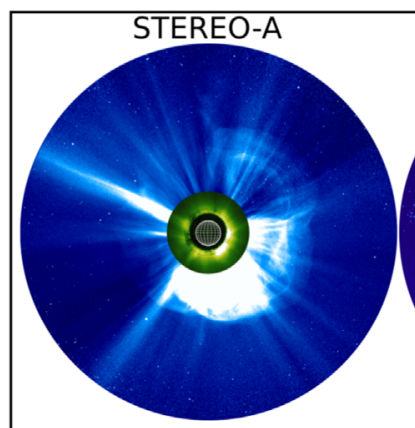

COR1+COR2 2012/07/23 03:24

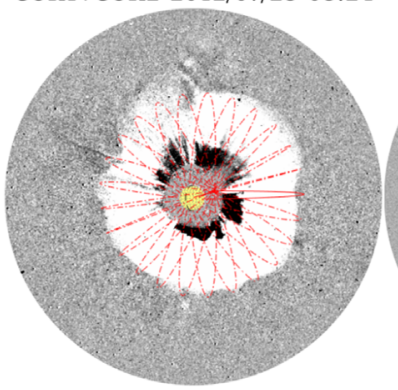

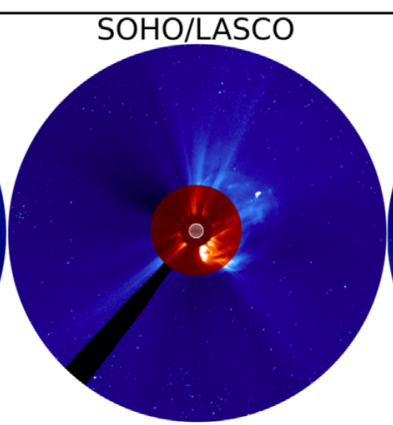

C2+C3 2012/07/23 03:30

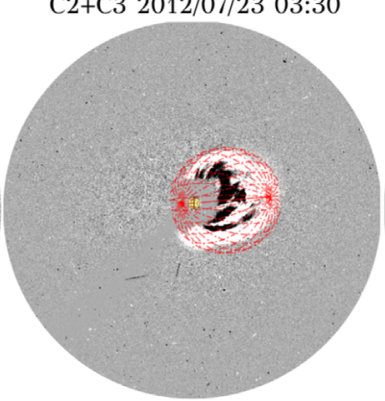

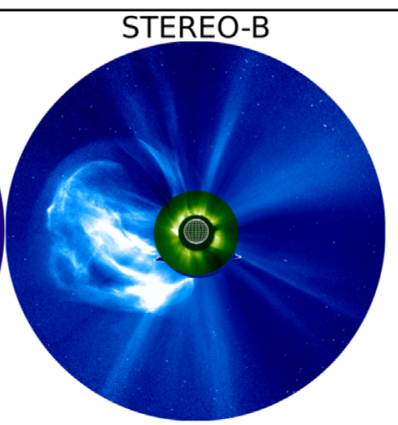

COR1+COR2 2012/07/23 03:24

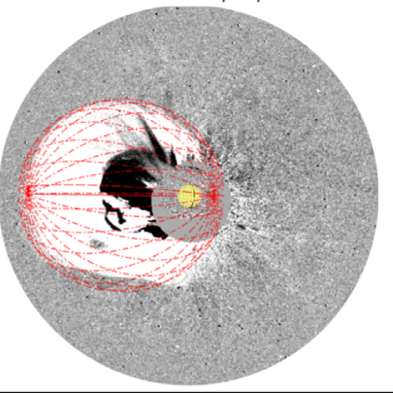

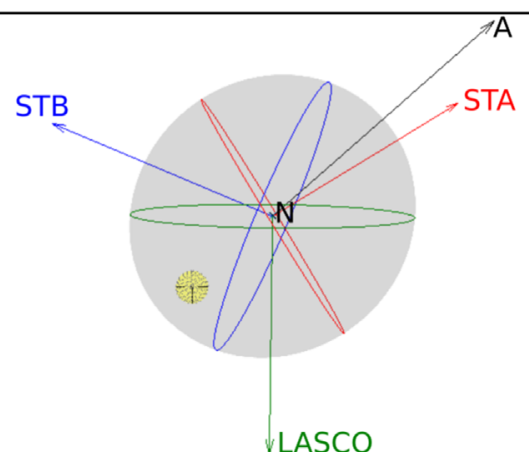

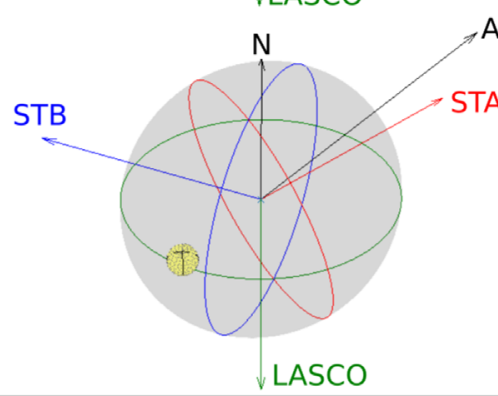

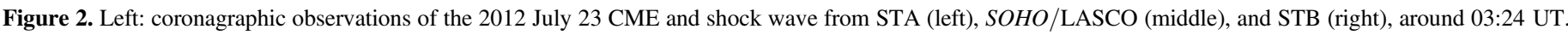

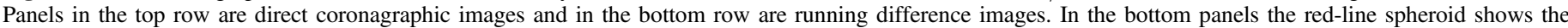

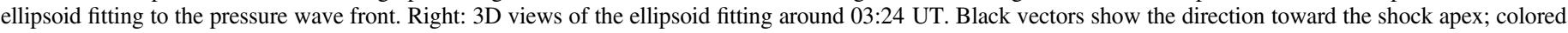
vectors show the direction of the different viewpoints. The colored ellipses label the tight constrained regions for each viewpoint.

passages of the interplanetary shock waves, we use solar wind measurements at Wind, ACE, and STEREO spacecraft from the OMNI database. ${ }^{12}$ Before the time of the shock passage in situ we seek and register the maximum intensity for each spacecraft.

\subsection{Deriving Shock Properties and Their Magnetic Connection}

\subsubsection{D Reconstruction Techniques}

Thanks to the nearly simultaneous white-light and EUV observations of pressure/shock waves, we can carry out the forward modeling of coronal pressure waves during all the SEP events on our list. In the low corona we use full-disk image triplets from the STEREO Extreme UltraViolet Imager (Wuelser et al. 2004) at $195 \AA$ and images from the Atmospheric Imaging Assembly (Lemen et al. 2012) on SDO at $193 \AA$. The field of view of the instruments extends to $\sim 1.7$ and $1.3 R_{\odot}$, respectively. Additionally, we use total brightness coronagraph image triplets from the Sun Earth Connection Coronal and Heliospheric Investigation (SECCHI; Howard et al. 2008) coronagraphs COR1 (FOV: $\sim 1.5-4 R_{\odot}$ ) and COR2 $\left(\sim 3-15 R_{\odot}\right)$ on board STEREO and the Large Angle and Spectrometric Coronagraph (LASCO; Brueckner et al. 1995) coronagraphs $\mathrm{C} 2\left(2.2-6 R_{\odot}\right)$ and $\mathrm{C} 3\left(3.7-30 R_{\odot}\right)$ on board $\mathrm{SOHO}$. To enhance the visibility of the waves in the EUV and coronagraphic data, we use running difference images when we perform the fittings.

To perform the 3D reconstruction of the observed pressure waves, we deploy a forward modeling technique that follows a similar approach to Thernisien et al. (2009), Kwon et al. (2014), and Rouillard et al. (2016). These methods are widely used to perform the geometrical fitting of shock waves or CMEs using two or three simultaneous viewpoints from

\footnotetext{
12 https://omniweb.gsfc.nasa.gov/
}

SECCHI and LASCO observations and employ different geometrical models (i.e., the spheroid or the graduated cylindrical model). In our analysis we are interested in 3D reconstructions of the pressure waves' frontal structure; we use an ellipsoid model and fit it at their outermost extent viewed from multiple vantage points. The geometrical shape and the position of the ellipsoid surface are defined, respectively, by a set of three parameters that control the length of the three axes of symmetry, which are pairwise perpendicular, and the center of the ellipsoid, which is defined in heliocentric coordinates. In the top panels of Figure 2 we show a set of coronagraphic observations for the 2012 July $23 \mathrm{CME}$ and its associated density wave. In the bottom panels we show the corresponding running difference images onto which we overlay the ellipsoid fitting to the pressure wave front, which is represented by the red lines. For this event, the 3D reconstructions of the pressure wave provided a maximum deprojected speed of $\sim 2700 \mathrm{~km} \mathrm{~s}^{-1}$ at the shock apex and $\sim 1850 \mathrm{~km} \mathrm{~s}^{-1}$ at the flanks.

The density/shock wave fitting procedure can be summarized as follows: (1) We start the fitting in the high corona (WL images) at times when coronagraphic images are temporally synchronized at the different spacecraft. (2) When the 3D fittings at these points have been performed, we then proceed at times with data from only one or two viewpoints. We make sure that the parameters that define the ellipsoids vary smoothly between optimal three-viewpoint fittings and those obtained from one/two viewpoints. (3) Finally, we address fittings in the low corona (EUV images), when the pressure wave forms, during which we often see near the base the formation of an EUV wave.

Once the ellipsoid fittings are performed, we apply a spline fitting to the ellipsoid parameters over time. With this method we reduce the variability of the parameters produced by random errors in the triangulation. This typically leads to a smooth displacement and expansion of the ellipsoid positions 
from one frame to another. From the spline fittings we produce a new set of ellipsoid parameters at steps of 1 minute to generate a final sequence of regularly time-spaced ellipsoids.

Application of the forward modeling technique with uninterrupted multiviewpoint observations results in very precise localization of the shocks at times when the STEREO and near-Earth spacecraft were equally separated around the Sun (such as from 2010 to 2012). Latitudinal and longitudinal shifts of as little as a degree in shock position are typically detected during the fitting. Different issues often affect the overall accuracy of the fittings from one event to another, such as the quality of the remote-sensing observations and the associated ability to identify and model accurately the same features in the different viewpoints. For the first type of uncertainties the accuracy of the fittings depends on (1) the general availability of simultaneous observations from at least three viewpoints, (2) the overall cadence of the imaging, (3) the existence of previous events and in general the overall contamination of the images (e.g., by high-energy particles), and (4) the separation angle of the viewpoints with respect to the source region. For items 1 and 2 the resulting uncertainty can be compensated by the smoothing of the ellipsoid parameters over time; for item 3 it is difficult to make robust fittings at high solar elongations when the features' contrast drops dramatically and the edges are not easily resolved especially in a contaminated background. However, this usually occurs above $\sim 20 R_{\odot}$, and beyond these heights the shock geometry departs from that of an ellipsoid and it is difficult to achieve a robust fitting. We therefore stop the shock modeling near $\sim 20 R_{\odot}$.

For item 4 the separation angle of the viewpoints may affect the accuracy of the fittings depending also on the source position. An example is given in Figure 2, where the green/ red/blue circles mark the regions on the ellipsoid that are tightly constrained from each viewpoint. They essentially map on the surface of the ellipsoid the locations along the line of sight where the line of sight is tangent to that surface. For this event the multiviewpoint imaging led to a robust fitting of both the radial and lateral expansion of the pressure wave. However, there are cases where it is nearly impossible to perform forward modeling with such accuracy because the viewing angles may not provide tight constraints in radial and lateral extension of the wave at the same time. The worst-case scenario is when the separation of all viewing pairs is significantly narrower than $\sim 20^{\circ}$. An example of this unfortunate case is the SEP event of 2014 September 25. In this event the CME has been observed in every viewpoint head-on (i.e., as a HALO-CME). Therefore, in this event only the lateral expansion of the shock wave can be modeled accurately since the edges of the shock apex are not captured in any of the viewpoints. We have discarded this event from our analysis. In no other events have we faced this problem.

Additionally, the expansion characteristics of the shock wave itself can affect the accuracy of the fittings. With the current geometrical fitting methods we can model robustly the large-scale envelope of the wave's frontal structure and also include in the models asymmetries like rotations or departures from the self-similar expansion. However, it is hard to model accurately small- or large-scale distortions to the modeled pressure waves. From the fittings of the pressure wave that we present in Figure 2 it is obvious that the fitted ellipsoid can capture accurately a large part of the observed WL pressure/ shock wave except from a region that is located at the south flank. This region is highly asymmetrical compared to the wave's overall frontal structure, and no treatment was here applied to improve the fitting in such regions. Despite these few difficulties faced with the simplicity of the 3D reconstruction, a rough estimate of the possible uncertainties in speed determination, for instance, gives errors of about $10 \%-20 \%$ in the toughest cases implied by poorer tie pointing and asymmetries.

\subsection{Deriving the Shocks' 3D Speed, Mach Numbers, and Compression Ratios}

To derive the 3D properties of the modeled coronal pressure/shock waves, we use the methods presented by Rouillard et al. (2016) and Plotnikov et al. (2017). We start with the sequence of the regularly time-spaced ellipsoids, and we consider a set of $71 \times 71$ grid points distributed over their surface. We compute the pressure waves' 3D expansion speed from the minimum distance between the grid points of two consecutive ellipsoids divided by their time interval, in our case $\delta t=1$ minute. This approach slightly underestimates the wave speed during the acceleration phase. For a mean acceleration of $500 \mathrm{~m} \mathrm{~s}^{-2}$ the difference would be $30 \mathrm{~km} \mathrm{~s}^{-1}$ in the time interval $\delta t$ of our consecutive computations, which yield a maximum error of $\sim 6 \%$ even for the slowest parts of the wave (i.e., for speed $\sim 500 \mathrm{~km} \mathrm{~s}^{-1}$ ). This error is negligible above $\sim 2-3 R_{\odot}$ when the acceleration phase has ended and the shock has reached a maximum or constant speed.

We compute the Mach numbers $\left(M_{\mathrm{A}}, M_{\mathrm{fm}}\right)$ and the magnetic field obliquity with respect to the shock normal $\left(\theta_{\mathrm{Bn}}\right)$, using the plasma and magnetic field properties provided from the Magneto-Hydrodynamic Around a Sphere Thermodynamic (MAST) model (Lionello et al. 2009). Those parameters are inferred along the surface of the pressure waves. The MAST model is an MHD model developed by Predictive Sciences Inc. ${ }^{13}$ that makes use of the photospheric magnetograms from $S D O / \mathrm{HMI}$ as the inner boundary condition of the magnetic field and includes detailed thermodynamics with realistic energy equations accounting for thermal conduction parallel to the magnetic field, radiative losses, and parameterized coronal heating. This thermodynamic MHD model produces more accurate estimates of plasma density and temperature in the corona (Lionello et al. 2009; Riley et al. 2011). The outer boundary of the model is at $30 R_{\odot}$. From the MAST 3D cubes we include at the front location the magnetic field and solar wind speed vector and the plasma temperature and density. The resolution of the MAST data cubes is $150 \times$ $100 \times 181$ in $r \times \theta \times \phi$ components. We calculate the Mach Alfvén $\left(M_{\mathrm{A}}\right)$, the fast magnetosonic Mach number $\left(M_{\mathrm{fm}}\right)$, and $\theta_{\mathrm{Bn}}$ from

$$
\begin{gathered}
\cos \theta_{\mathrm{BN}}=\frac{\boldsymbol{B} \cdot \hat{\boldsymbol{n}}}{|\boldsymbol{B}|}, \\
M_{\mathrm{A}}=\frac{V_{\mathrm{sh}}-\boldsymbol{V}_{\mathrm{sW}} \cdot \hat{\boldsymbol{n}}}{V_{\mathrm{A}}}, \\
M_{\mathrm{fm}}=\frac{V_{\mathrm{sh}}-V_{\mathrm{sw}} \cdot \hat{\boldsymbol{n}}}{V_{\mathrm{fm}}},
\end{gathered}
$$

where $\hat{\boldsymbol{n}}$ is the shock-normal vector, $V_{\mathrm{sh}}$ is the shock speed, $V_{\mathrm{A}}$ is the Alfvén speed, $V_{\mathrm{fm}}$ is the fast magnetosonic speed, and $V_{\mathrm{sw}}$ is the background solar wind speed vector. Our

\footnotetext{
${ }^{13}$ http://www.predsci.com/
} 
calculations are in the local solar wind frame (see Section 4 in Plotnikov et al. 2017, for more details). Before we proceed with the calculation of the shock properties from the 3D shock fittings and the MAST model, we apply a calibration of the 3D MHD magnetic field and density data using in situ measurements. For the 3D MHD magnetic field data, Rouillard et al. (2016) showed that the average radial field measured at the outer boundary of the MAST model (and also in the potential field source surface (PFSS) model) is lower than the measured radial field values near 1 au; therefore, a calibration (correction factor) is necessary. The correction factor for the magnetic field can be obtained by comparing the radial magnetic field components of the MHD cubes at an outer boundary with the values measured near $1 \mathrm{au}$. From the MHD models we calculate the average of the unsigned radial field component over an entire source surface and apply an inverse square dependence to compare with in situ measurements near $1 \mathrm{au}$. The height of the source surface is defined as the distance where the magnetic field lines are mostly open to the interplanetary medium and magnetic fields have become very radial. In our analysis we estimate the correction factor using different heights for the surface situated between 1 and $15 R_{\odot}$. The distance of the source surface where the correction factor starts to have a constant value is on average located near $\sim 2.5-3.5 R_{\odot}$, hence very close to the source surface of the PFSS model.

To derive the magnetic correction factors, we process the in situ measurements in the same manner as in Rouillard et al. (2016) to compare them with the extrapolated average values from the MHD data (also see Rouillard et al. 2007). We determine the absolute magnetic field values considering a full solar rotation period and using magnetic field measurements from the OMNI database and the STEREO spacecraft. To preserve the global topology of the field, we apply the correction factor to both open- and closed-field regions of the corona. However, the closed-field regions cannot be related to the in situ measurements made near 1 au. Since we are interested in the SEP propagation along open magnetic field lines to $1 \mathrm{au}$, we adopt this technique. From the overall analysis of the magnetic correction factors of the MHD cubes we have that their values vary from $\sim 1.6$ to $\sim 2.4$ with an average of $\sim 1.8$. Since the Alfvén speed is proportional to the ambient magnetic field strength, the correction of magnetic field will have for effect to decrease the computed Mach numbers of the shock, thereby providing low estimates.

The plasma density structures simulated by the MAST model are capable of reproducing global coronal features observed in WL, EUV, and X-ray emission (see Lionello et al. 2009; Rušin et al. 2010). Systematic comparisons over several solar rotations between the simulated coronal density and the density from the inversion of polarized brightness WL observations have shown that the density distributions are consistent overall with each other within a factor of $1 / 2$ to 2 (e.g., de Patoul et al. 2015; Wang et al. 2017). To derive the density correction factors, we follow the same procedure as the one described above to derive the magnetic correction factors. We average the density values of the MAST data over the an entire source surface at different distances, and we extrapolate those values at $1 \mathrm{au}$. This region was situated beyond the region where the solar wind forms, i.e., at the very least $>20 R_{\odot}$. Then, we compare the derived densities with the average densities calculated from the in situ observations over a complete solar rotation. To make our calculations more robust, we used in situ solar wind measurements from STA, STB, and L1. We found that the density correction factors vary from $\sim 0.30$ to $\sim 0.63$, with an average value of $\sim 0.45$.

From the determined values of the density correction factors it is evident that the MAST model may systematically overestimate the densities when we extrapolate the simulated values to $1 \mathrm{au}$ and compare them with the in situ measurements. However, it is worth noting that the intercomparisons between the observed and the simulated densities might be in reality more complex. Wang et al. (2017) presented in their Figure 7 a comparison between the 3D coronal electron densities derived by the spherically symmetric polynomial approximation (SSPA) inversion method using remote-sensing observations from STEREO coronagraphs and the MHD simulated densities. The density ratio between the MAST model and SSPA inversion method ranges from $1 / 8$ to 5 at heights between 2.0 and $3.0 R_{\odot}$. We note that the coronal brightness below $3.0 R_{\odot}$ is mainly controlled by closed-field regions; however, in our analysis what matters is the open field, and therefore the coronal brightness beyond the region where the solar wind forms.

Therefore, if we neglect for the moment where those differences arise, it is not certain whether the simulated densities overestimate or underestimate the "true densities" (if we could measure them in situ). Those differences could be attributed either to the WL image inversion methods or to the MAST simulation. This is an interesting question that could be addressed when PSP returns its first in situ measurements below $30 R_{\odot}$.

Considering these uncertainties, we assume in our analysis that the simulated densities are accurate within $1 / 2$ to 2 times the "true coronal densities," and we will apply the density correction factors as a separate step to our statistical analysis. We note that since the Alfvén speed is inversely dependent on the square root of the plasma density, the correction of density will have the effect of decreasing the computed Mach numbers of the shock, thereby providing conservative estimates.

Using the method presented in A. Kouloumvakos et. al. (2019, in preparation), we calculate the density compression ratio $(X)$ from the explicit solutions of Rankine-Hugoniot (R$\mathrm{H})$ jump conditions for MHD and the plasma and magnetic field properties derived at the front location. From the R-H jump conditions we derive a third-degree polynomial of the compression ratio as a function of the $M_{\mathrm{A}}$, plasma beta, and $\theta_{\mathrm{Bn}}$. We assume that the polytropic indices upstream and downstream from the shock are the same (see Section 3 in Livadiotis 2015). The solutions of the cubic trinomial can be obtained analytically and are given by Equation (25) $\alpha$ in Livadiotis (2015). We note that these three solutions essentially describe the compressional fast- and slow-mode MHD waves and/or intermediate waves. Additionally, from the derived density compression ratios we also calculate the magnetic compression ratio (MR) again from the explicit solutions of $\mathrm{R}-\mathrm{H}$ conditions and the scattering center compression ratio (rc) from the relation $r_{c}=X\left(1-\cos \theta_{\mathrm{Bn}} M_{\mathrm{A}}^{-1}\right)$ (see Vainio et al. 2014; Afanasiev et al. 2018).

In Figure 3 we show a 3D view of the reconstructed pressure wave for the event of 2014 February 25. Panels (a)-(d) present the $3 \mathrm{D}$ distributions of different parameters, i.e., the speed, 

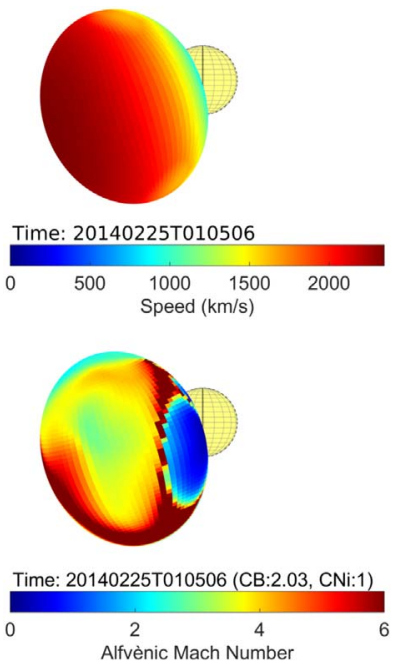

Figure 3. Result of the 3D properties along the reconstructed pressure wave front surface for the 2014 February 25 event around 01:05 UT. Panels show (a) the wave speed, (b) the magnetic field orientation with respect to the perpendicular direction to the front surface $\left(\theta_{\mathrm{Bn}}\right)$, (c) the Alfvénic Mach number $\left(M_{\mathrm{A}}\right)$, and $(\mathrm{d})$ the density compression ratio.

$\theta_{\mathrm{Bn}}, M_{\mathrm{A}}$, and $X$ that are depicted with overplotted color maps along the ellipsoid surface. The pressure wave speed varies from $\sim 2100 \mathrm{~km} \mathrm{~s}^{-1}$ at the apex to $\sim 1500 \mathrm{~km} \mathrm{~s}^{-1}$ on the flank (see panel (a)). The geometry also changes from quasi-parallel to quasi-perpendicular, as expected, in the same regions. The $M_{\mathrm{A}}$ values range from $\sim 0.5$ to $\sim 8$, with the highest values measured in the vicinity of the neutral line, located for this particular event near the western flank and mostly over a broad region south of the apex. There is also a region in the west flank near the neutral line with $M_{\mathrm{A}}$ values lower than $\sim 2$. In this region it is unlikely that a strong shock wave formed. This is more apparent in panel (d) of Figure 3, where we show the 3D density compression ratio. The low values of $M_{\mathrm{A}}$ and possibly the geometry do not give density compression ratios higher than 1 for the solutions of the R-H conditions; therefore, these regions are unlikely to produce a shock. The compression ratio ranges between $\sim 3$ and 4 in the region from the shock flanks to the apex.

\subsection{Shock Parameters at Magnetically Well-connected Regions}

To compare the properties of energetic particles measured near $1 \mathrm{au}$ with the 3D properties of shocks inferred in the corona, we must consider the magnetic field lines that connect our reconstructed shock front surface with the points of in situ measurements. Using the magnetic field vector data from the MAST 3D cubes, we perform a field line tracing starting at the wave front and antisunward until the outer boundary of MAST simulation domain $\left(30 R_{\odot}\right)$. In this paper we consider only the open-field lines that intersect the reconstructed front surface and connect in situ spacecraft. The field line tracing is performed at every time step between launch until $\sim 90$ minutes into the event.

Some weak points of using the MAST data to trace magnetic field lines are low grid resolutions above $10 R_{\odot}$ and the numerical diffusion. The resulting uncertainties in field line tracing can be estimated by considering the intersection point between the Parker spiral and the traced field lines at heights below $10 R_{\odot}$. The top panels of Figure 4 show a 3D view of the resulting magnetic field line tracing for the 2014 February 25 event, around 01:05 UT. Here we present 20\% of the 5041 open and closed magnetic field lines that we traced. In panels (a)-(c), the different colors for the traced field lines are defined by the shock parameters at the point of intersection of the field lines with the wave surface.

A way to sample the regional and temporal variability of shock parameters near the shock locations that are magnetically connected to in situ detectors is to map this variability on latitude-longitude maps or "Carrington maps." For a particular height in the solar atmosphere (that defines a sphere) we compute the latitude and longitude of each field line connected to the shock. We then display on that map the parameters of the shock wave at the intersection point of the field line and the shock. Essentially, the maps we use here are similar to those introduced by Plotnikov et al. (2017) to study solar gamma-ray events. With this representation it is more straightforward to evaluate how uncertainties in magnetic connectivity may affect our comparison of shock properties with SEPs measured at specific points in the inner heliosphere. Examples of the produced maps are given in the middle panels of Figure 4 at a distance of $5 R_{\odot}$ and a bin size of $20^{\circ} \times 15^{\circ}$ in longitude and latitude, respectively. From left to right we show in the middle panels of Figure 4 the average values of the shock speed, $M_{\mathrm{A}}$, and density compression ratio, respectively.

In this study we use a bin size of $20^{\circ} \times 15^{\circ}$ when we perform the statistical analysis, and we examine the correlations in Section 3. Considering the uncertainty faced to establish magnetic connections with the instruments, the bin size we will use provides a good upper limit. In terms of the uncertainty in connectivity induced by the variability of the solar wind speed, the selected bin size corresponds to an uncertainty of $\sim 150-200 \mathrm{~km} \mathrm{~s}^{-1}$. Additionally, a bin size of $20^{\circ} \times 15^{\circ}$ provides a good statistical sample in every region in the map. We performed some further tests to validate these analysis uncertainties. Lower bin sizes up to $\sim 15^{\circ} \times 10^{\circ}$ gave us results that were qualitatively and quantitatively similar.

As a next step we use these maps to derive the temporal evolution of the shock parameters only at the magnetically well-connected regions with the instruments at 1 au. First, we assume that the interplanetary field lines can be roughly modeled as an Archimedian spiral, and we calculate the location of the magnetically well-connected footpoints for each spacecraft. The locus of the spirals and therefore the location of the footpoints of the field lines of interest are controlled by the speed of the solar wind carrying them and the solar rotation period. We use the average solar wind speed measured in situ near the onset time of the SEP event. The assumption of an Archimedian spiral to connect near-1 au observations with the shock requires that the SEP event occurred during quiet solar wind conditions in the region situated between the Sun and the instrument making the in situ measurements. This might not be the case for SEP events that occur at periods of extreme solar activity. We overplot on the maps the location of the spiral footpoint for each spacecraft (see Figure 4, middle row); to select the bins, we perform the statistics of the shock parameter. An example of the temporal evolution of the shock parameters, at the well-connected regions for each spacecraft, is given in the bottom panels of Figure 4.

The uncertainty of the derived shock parameters at the magnetically well-connected field lines is evaluated from the parameters' statistics. The upper and lower limits are derived 

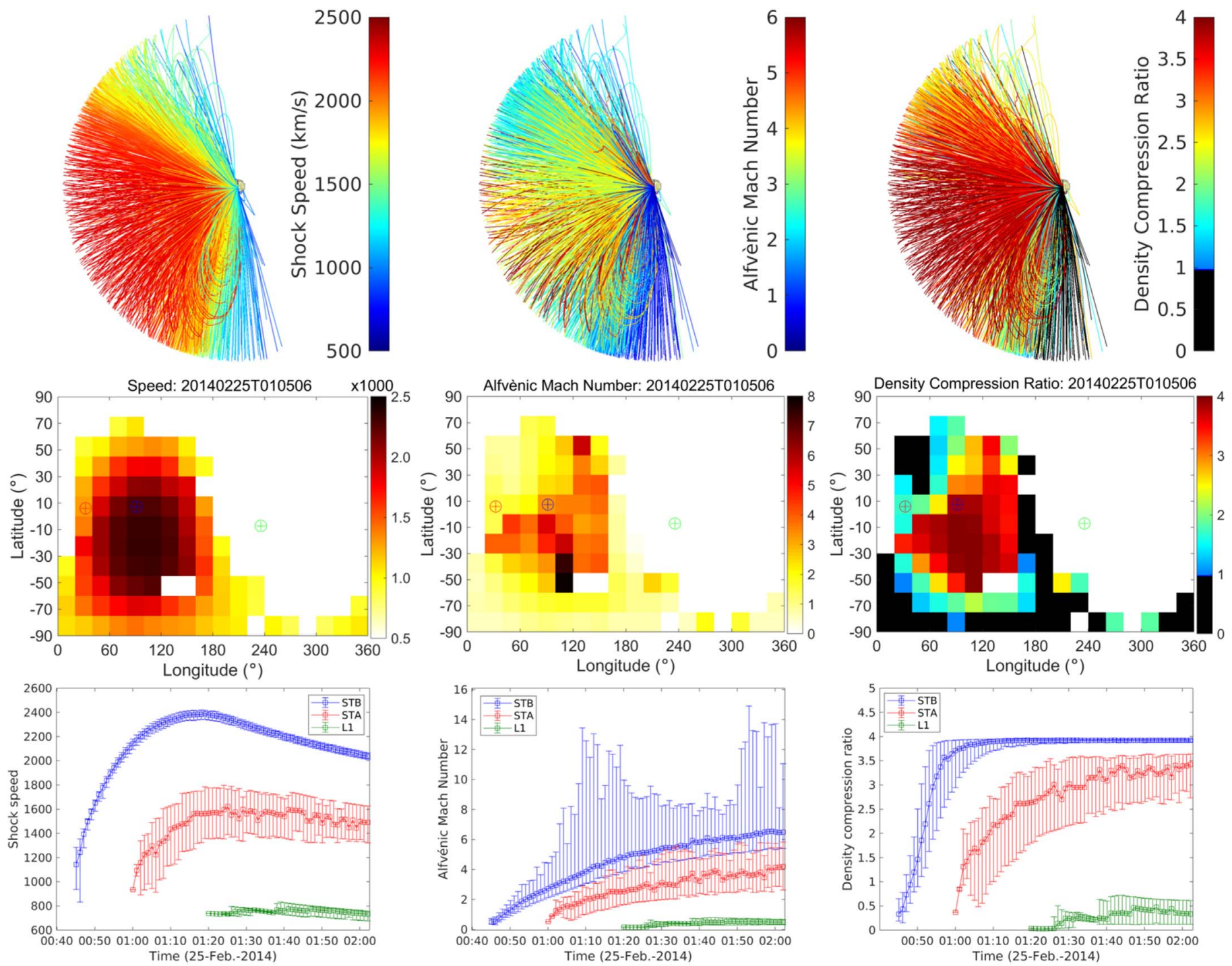

Figure 4. Top: 3D view of the magnetic field lines that connect to the shock surface, for the 2014 February 25 event around 01:05 UT. The field lines are color-coded by the different shock parameters, the shock speed, $M_{\mathrm{A}}$, and density compression ratio from left to right panels, respectively. Middle: binned "Carrington maps" at a distance of $5 R_{\odot}$ showing the average shock parameters registered to the open magnetic field lines connected to the modeled shock wave surface (see the text for details). The map's bin size is $20^{\circ} \times 15^{\circ}$ in longitude and latitude. The overplotted colored points show the location of the spiral footpoint for each viewpoint: green for Earth, blue for STB, and red for STA. Bottom: temporal evolution of the shock parameters at the well-connected regions for each viewpoint.

from the distributions' values at the 10th and 90th percentiles. We use those estimates of the shock parameter uncertainties in the next sections, where we compare the modeled shock parameters with SEP measurements.

\section{Relating the SEP Properties to Shock Parameters}

\subsection{Correlation of SEP $\mathrm{I}_{\text {peak }}$ with Shock Speed}

We start this analysis with the correlation of the SEP peak intensities at 20-26 MeV (Ip20), 40-60 MeV (Ip40), and 60-100 MeV (Ip60) with the shock speed (see Sections 2.2 and 2.4). We use throughout our analysis logarithms when we perform regressions and correlations because of the dynamic range of the peak intensities and the other parameters. The log $-\log$ regression fittings yield the following relation between our measurements: Ip $\sim k_{2}^{\prime} V^{k_{1}^{\prime}}$. The coefficients can be directly comparable to those derived in previous studies (e.g., Kahler \& Vourlidas 2013). To determine the correlation between the parameters, we use the Pearson's correlation coefficient (CC), which measures the amount of linear dependence between two variables, and it is defined as the covariance of the two variables divided by the product of their standard deviations.

The top row of Figure 5 shows the correlation between the Ip and the maximum speed derived at the shock apex during its evolution, for the three energy bands we consider for this study. Here, we consider only S/C-specific SEP events with a magnetic connection longitude that is less than $50^{\circ}$. We also show the same correlations with the projected CME speed. As already discussed in Section 2.1, to account for (1) the random errors in the estimation of the peak intensities and (2) any errors produced from the calibration of the different instruments at the energy channels, we use for the uncertainty in Ip a standard error of $50 \%$ of their measured values. Additionally, for the uncertainty of the determined shock speed at the apex we use a standard error of $10 \%$, which is a rough estimate based on the accuracy of 3D ellipsoid fittings (see Section 2.3.1). We use the same error for the CME speed; however, we recognize that this might be greater. 

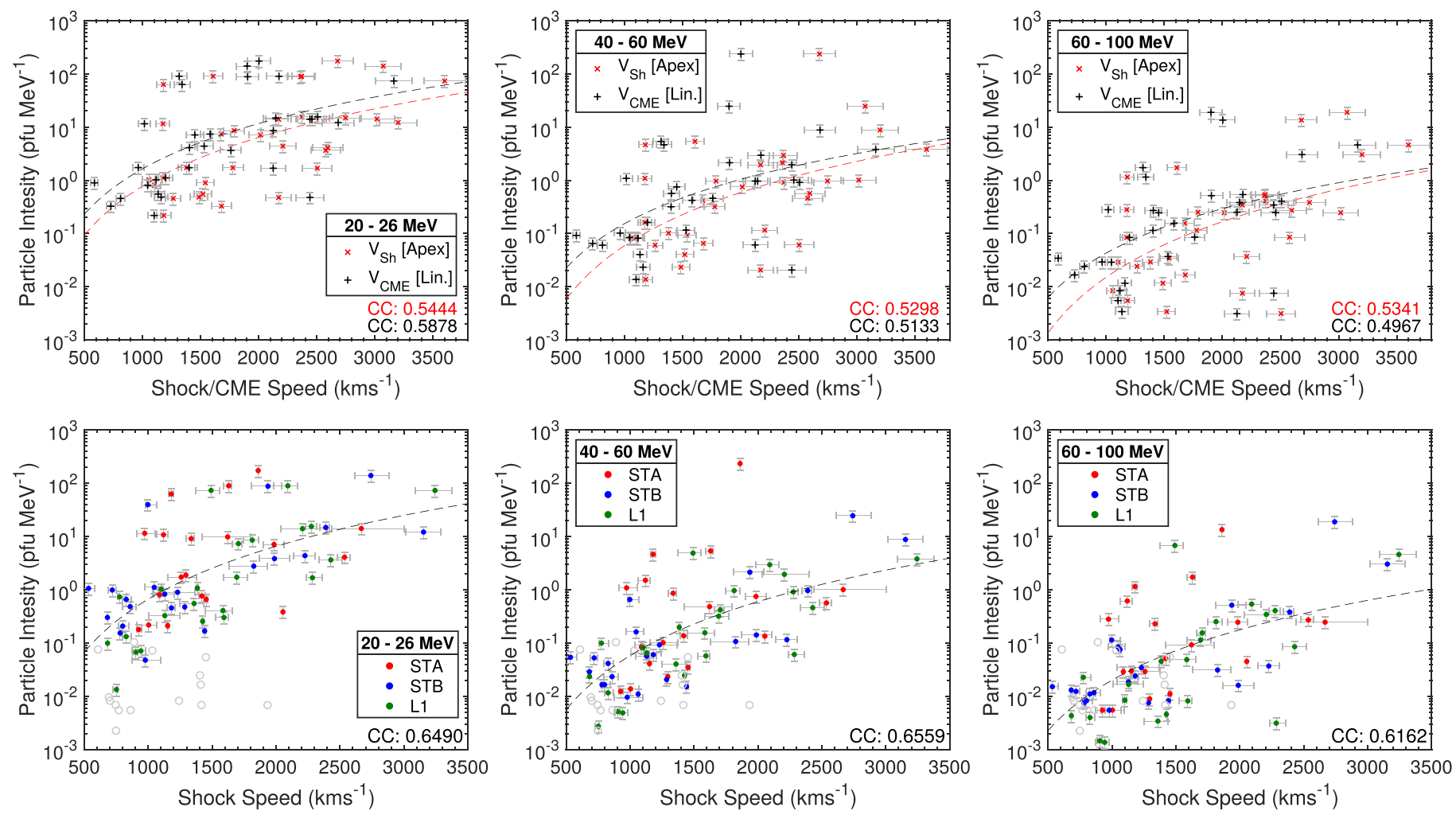

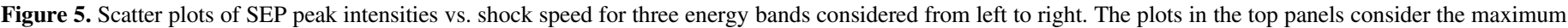

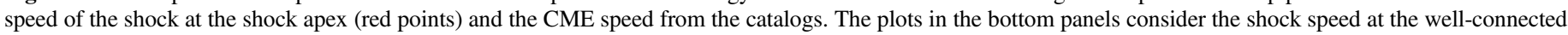

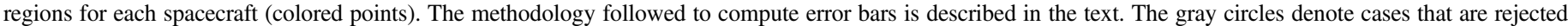
from the analysis (see the text for details). The dashed lines are power-law fittings to the measurements.

From the Pearson's correlation between the Ip and the shock speeds at the apex we found that the CCs hardly exceed 55\% in every case, despite the fact that the speeds do not suffer from projection effects since they are derived from the shock fittings. The highest CC is observed for Ip20, CC $=54.4 \%$. As noted in Section 2.3.1, for our analysis we use the speeds that are directly derived from the 3D shock fittings and not from measurements in the plane of the sky. The lack of a good correlation is more or less expected since the speed at the shock apex is unlikely to be, for most events, a representative parameter of the region at which the particles are accelerated. A first expectation is that when the shock parameters are derived directly at the well-connected field lines, the correlations will improve significantly. Additionally, we found similar CC values for the $\mathrm{CME}$ speeds. The best $\mathrm{CC}$ is observed for Ip20, $\mathrm{CC}=58.8 \%$.

In the bottom row of Figure 5 we present the correlation of Ip with the maximum shock speed determined at the wellconnected regions. We exclude from the correlations the events where the SEP onset times are registered $>3-6 \mathrm{hr}$ later (depending on the energy) than the time when shock waves pass the magnetically well-connected regions. For those SEPs, the the link between shock wave expansion and the timing of SEPs may be affected by transport processes. We examine those cases in more detail in Section 3.6. From here on, we will use gray circles to depict those cases that are rejected from our statistical analysis.

From the CCs of Figure 5 (bottom row) it is clear that we have a significant improvement of the CCs and their $p$-values in every energy range, when we compare Ip with the shock speed at the well-connected regions (i.e., when we included the magnetic connectivities to our analysis). The best correlation is observed for Ip20 with $\mathrm{CC}_{\mathrm{V} 20}=64.9 \%$, while for Ip40 and Ip60 we have $\mathrm{CC}_{\mathrm{V} 40}=65.6 \%$ and $\mathrm{CC}_{\mathrm{V} 60}=61.6 \%$, respectively. The calculated $p$-values for our correlations are on the order of $10^{-8}-10^{-9}$, so the CCs are statistically significant. The aggregate results for the derived Pearson's CCs and their $p$-values are summarized in Table 2. From the fitted function, $\log \left(I_{p}\right)=k_{1} \log (V)+k_{2}$, we have the following coefficients, $k=\left[k_{1}, k_{2}\right]: k=[3.30,-23.19]$ for Ip20, $k=[3.36,-26.06]$ for Ip40, and $k=[3.14,-25.60]$ for Ip60.

Previous studies have shown that there is a slight tendency for the CCs of Ip with shock speed to decrease at higher energies (see Papaioannou et al. 2016). This trend appears in our analysis, but it is too small an effect to tell whether this dependence is statistically important. We can evaluate how significant it is by examining the confidence interval of the Pearson's coefficient using the so-called Fisher $r$-to- $z$ transformation. This method has been applied in statistical analysis of SEP characteristics by Papaioannou et al. (2016) and cosmic rays (see also Rouillard \& Lockwood 2004). Essentially, for a specified value of a confidence interval, it is possible to calculate the limits of the confidence interval around our CCs from the Fisher transformation. This transformation is defined as $z_{r}=\operatorname{artanh}(r)$, where $r$ is the Pearson's CC. For a confidence interval of $90 \%$ (significance level: $\alpha=0.1$ ) we determine upper and lower limits of our CCs using the relation $r_{+/-}=\tanh \left(z_{u / l}\right)=\tanh \left(z_{r} \pm z_{1-\alpha / 2} \sigma_{z}\right)$, where $z_{1-\alpha / 2}=100(1-a / 2), \sigma_{z}$ is the standard deviation $\sigma_{z}=1 / \sqrt{N-3}$ and $N$ is the sample size used for the CC determination. In Table 2 we include the calculated upper and lower limits for the CCs. From the small differences between the $\mathrm{CCs}$ at the different energies and the width of the calculated 
Table 2

Pearson's Correlation Coefficients between the SEP Peak Fluxes in Three Energy Channels and the Different Shock Parameters

\begin{tabular}{|c|c|c|c|}
\hline $\begin{array}{l}\mathrm{CCs} \\
(1)\end{array}$ & $\begin{array}{l}\text { Ip20 } \\
(2)\end{array}$ & $\begin{array}{l}\text { Ip40 } \\
\text { (3) }\end{array}$ & $\begin{array}{l}\text { Ip60 } \\
(4)\end{array}$ \\
\hline$V_{\mathrm{CME}}$ & $\begin{array}{c}58.8 \%\left(10^{-4}\right) \\
{[41 \%, 72 \%]}\end{array}$ & $\begin{array}{c}51.3 \%(0.002) \\
{[33 \%, 68 \%]}\end{array}$ & $\begin{array}{c}49.7 \%(0.003) \\
{[30 \%, 66 \%]}\end{array}$ \\
\hline$V_{\mathrm{sh}}^{\mathrm{a}}$ & $\begin{array}{c}54.4 \%(0.001) \\
{[36 \%, 69 \%]}\end{array}$ & $\begin{array}{c}53.0 \%(0.002) \\
{[34 \%, 68 \%]}\end{array}$ & $\begin{array}{c}53.4 \%(0.002) \\
{[33 \%, 68 \%]}\end{array}$ \\
\hline$V_{\mathrm{sh}}$ & $\begin{array}{c}64.9 \%\left(10^{-9}\right) \\
{[56 \%, 74 \%]}\end{array}$ & $\begin{array}{c}65.6 \%\left(10^{-9}\right) \\
{[56 \%, 74 \%]}\end{array}$ & $\begin{array}{c}61.6 \%\left(10^{-8}\right) \\
{[51 \%, 71 \%]}\end{array}$ \\
\hline$M_{\mathrm{A}}$ & $\begin{array}{c}71.4 \%\left(10^{-11}\right) \\
{[63 \%, 79 \%]}\end{array}$ & $\begin{array}{c}75.6 \%\left(10^{-13}\right) \\
{[68 \%, 82 \%]}\end{array}$ & $\begin{array}{c}68.9 \%\left(10^{-10}\right) \\
{[59 \%, 77 \%]}\end{array}$ \\
\hline$M_{\mathrm{A}}^{\mathrm{b}}$ & $\begin{array}{c}71.4 \%\left(10^{-11}\right) \\
{[63 \%, 79 \%]}\end{array}$ & $\begin{array}{c}75.0 \%\left(10^{-13}\right) \\
{[67 \%, 81 \%]}\end{array}$ & $\begin{array}{c}68.6 \%\left(10^{-10}\right) \\
{[59 \%, 76 \%]}\end{array}$ \\
\hline$M_{\mathrm{fm}}$ & $\begin{array}{c}67.7 \%\left(10^{-10}\right) \\
{[59 \%, 76 \%]}\end{array}$ & $\begin{array}{c}69.7 \%\left(10^{-11}\right) \\
{[61 \%, 77 \%]}\end{array}$ & $\begin{array}{c}64.3 \%\left(10^{-9}\right) \\
{[54 \%, 73 \%]}\end{array}$ \\
\hline$M_{\mathrm{fm}}{ }^{\mathrm{b}}$ & $\begin{array}{c}67.6 \%\left(10^{-10}\right) \\
{[59 \%, 76 \%]}\end{array}$ & $\begin{array}{c}69.1 \%\left(10^{-10}\right) \\
{[60 \%, 77 \%]}\end{array}$ & $\begin{array}{c}63.9 \%\left(10^{-9}\right) \\
{[54 \%, 73 \%]}\end{array}$ \\
\hline$X$ & $\begin{array}{c}60.4 \%\left(10^{-8}\right) \\
{[50 \%, 70 \%]}\end{array}$ & $\begin{array}{c}59.4 \%\left(10^{-7}\right) \\
{[48 \%, 69 \%]}\end{array}$ & $\begin{array}{c}53.4 \%\left(10^{-6}\right) \\
{[41 \%, 64 \%]}\end{array}$ \\
\hline MR & $\begin{array}{c}50.3 \%\left(10^{-5}\right) \\
{[38 \%, 62 \%]}\end{array}$ & $\begin{array}{c}55.0 \%\left(10^{-6}\right) \\
{[42 \%, 65 \%]}\end{array}$ & $\begin{array}{c}47.4 \%\left(10^{-5}\right) \\
{[33 \%, 58 \%]}\end{array}$ \\
\hline $\mathrm{rc}$ & $\begin{array}{c}55.8 \%\left(10^{-6}\right) \\
{[46 \%, 69 \%]}\end{array}$ & $\begin{array}{c}62.0 \%\left(10^{-7}\right) \\
{[53 \%, 72 \%]}\end{array}$ & $\begin{array}{c}53.3 \%\left(10^{-6}\right) \\
{[45 \%, 67 \%]}\end{array}$ \\
\hline$\theta_{\mathrm{Bn}}^{M}$ & $\begin{array}{c}-15.7 \%(0.15) \\
{[-33 \%, 0 \%]}\end{array}$ & $\begin{array}{c}-10.5 \%(0.3) \\
{[-27 \%,-1 \%]}\end{array}$ & $\begin{array}{c}-12.3 \%(0.3) \\
{[-29 \%, 0 \%]}\end{array}$ \\
\hline$\theta_{\mathrm{Bn}}^{C}$ & $\begin{array}{l}26.8 \%(0.14) \\
{[0 \%,-45 \%]}\end{array}$ & $\begin{array}{c}28.1 \%(0.1) \\
{[1 \%, 47 \%]}\end{array}$ & $\begin{array}{c}30.0 \%(0.1) \\
{[0 \%, 47 \%]}\end{array}$ \\
\hline
\end{tabular}

Notes. Next to the Pearson's correlation coefficients we include the $p$-value of the statistics, and below we give in parentheses the upper and lower bounds of the CCs from the Fisher transformation, calculated for a confidence interval of $90 \%$.

a Maximum shock speed measured at the apex.

${ }^{\mathrm{b}}$ With the application of the density correction factors.

bounds from the Fisher transformation it is rather inconclusive whether the speed-Ip correlations have any energy dependence.

\subsection{Correlation of SEP $\mathrm{I}_{\text {peak }}$ with Shock Mach Numbers}

Next, we examine the correlation between the SEP peak intensities and the maximum Alfvénic or fast magnetosonic Mach number, both determined at the well-connected field lines. A first expectation is that the correlations should be significant based on the results of Reames (2012) and Rouillard et al. (2016). Additionally, recent simulations of DSA (e.g., Afanasiev et al. 2018) show that the acceleration efficiency of the shock tends to be higher for larger values of the scattering center compression ratio that depends on $M_{\mathrm{A}}$.

In Figure 6 we show the correlations between Ip20, Ip40, Ip60, and $M_{\mathrm{A}}$. The uncertainty (i.e., the error bars in Figure 6) of the derived $M_{\mathrm{A}}$ values is evaluated from the statistics on the well-connected field lines, and for the Ip we use a standard error of $50 \%$ as explained earlier in the text. In the top panels of Figure 6 we show the correlations between the SEPs' peak intensity and $M_{\mathrm{A}}$ without considering the density correction factors (see Section 2.4 for details). In the bottom panels we show the same correlations with the application of the density correction. The $\mathrm{CCs}$ remain almost unchanged after the application of the density correction factors. The correction of the simulated densities acts as a scale factor for the $M_{\mathrm{A}}$ values and has no effect on the statistical output.
From the correlations of Figure 6 we find that $M_{\mathrm{A}}$ correlate extremely well with Ip in all three energy ranges, with CCs greater than $70 \%$. Essentially, the resulting relations and correlations show that the magnetically well-connected shock regions that have high Alfvénic Mach number relate well to SEP events observed in situ with high peak intensities and vice versa. This is an important result that shows a direct connection of the properties of the shock region that the energetic particles accelerated and released into the open magnetic field lines and the characteristics of the SEPs measured in situ in terms of their peak intensity. Another aspect that might be of importance arises from the rejected points (see Section 3.1), which we depict with the gray circles in Figure 6. In all cases except for some outliers (two to four cases depending on the energy) the points concentrate in a region of low peak intensities and $M_{\mathrm{A}}$ less than $\sim 2$, while almost half of rejected cases (e.g., 7/15 for 40-60 MeV) have $M_{\mathrm{A}}$ less than one for which a shock was unlikely to form along the connected magnetic field lines (see also Section 3.6). From the fitted function, $\log \left(I_{p}\right)=k_{1} \log \left(M_{\mathrm{A}}\right)+k_{2}$, we have the following coefficients: $k=[1.94,-1.81]$ for Ip20, $k=[2.07$, -4.38] for Ip40, and $k=[1.88,-5.29]$ for Ip60.

The best correlation is found for Ip40, $\mathrm{CC}_{\mathrm{MA} 40}=75.6 \%$. We find smaller correlations for Ip20, $\mathrm{CC}_{\mathrm{MA} 20}=71.4 \%$, and for $\mathrm{Ip} 60, \mathrm{CC}_{\mathrm{MA} 60}=68.9 \%$. Additionally, the calculated $p$ values for the $\mathrm{CCs}$ are on the order of $10^{-10}-10^{-13}$ (see Table 2). We also calculate the $\mathrm{CC}$ bounds from the Fisher transformation in Table 2. Comparing the correlations between $M_{\mathrm{A}}$ and the shock speed with the Ip, it seems that the CCs are significantly improved for $M_{\mathrm{A}}$. The relative improvement of the CCs ranges from $\sim 6 \%$ to $10 \%$, depending on the energy. Considering the upper and lower bounds of the CCs from the Fisher transformation, we evaluate the significance of the difference between the $\mathrm{CC}_{V}$ and $\mathrm{CC}_{\mathrm{MA}}$. The derived difference probabilities range between $\sim 0.13$ and $\sim 0.23$ (i.e., confidence $87 \%-76 \%$ ). For the $\mathrm{CC}_{\mathrm{V} 40}$ and $\mathrm{CC}_{\mathrm{MA} 40}$ we find that there is a significant difference with a confidence of $\sim 87 \%$, while for the $\mathrm{CCs}$ at the other two energy ranges considered here, the nullhypothesis test gives lower confidence intervals. It is rather inconclusive whether the difference is significant or not in those cases. This is likely an effect of the low statistical sample that we have (65-70 points depending on the energy). Additionally, it is not clear in our statistics whether there is an energy dependence on the CCs; however, the weaker correlation is found for the high-energy channel, 60-100 MeV.

In Figure 7 we show the correlations between the SEPs' peak intensities (Ip20, Ip40, Ip60) and the fast magnetosonic Mach number, $M_{\mathrm{fm}}$. Comparing the $\mathrm{CCs}$ of $\mathrm{CC}_{\mathrm{Mfm}}$ with the shock speed correlations, we find a relative improvement of $\sim 4 \%$. Again the resulting $\mathrm{CC}$ values are similar either with the application of the density correction factors or not (see Table 2); however, the CCs are lower than those found for $M_{\mathrm{A}}$.

\subsection{Correlation of SEP $\mathrm{I}_{\text {peak }}$ with Shock Compression Ratios}

In Figure 8 we present the correlations of Ip with the shocks' compression ratios. From top to bottom we show the density $(X)$, the MR, and the scattering center (rc) compression ratio, ${ }^{14}$

\footnotetext{
${ }^{14}$ We note that we have calculated the scattering center compression ratio assuming that the downstream scattering center speed is equal to the fluid speed.
} 

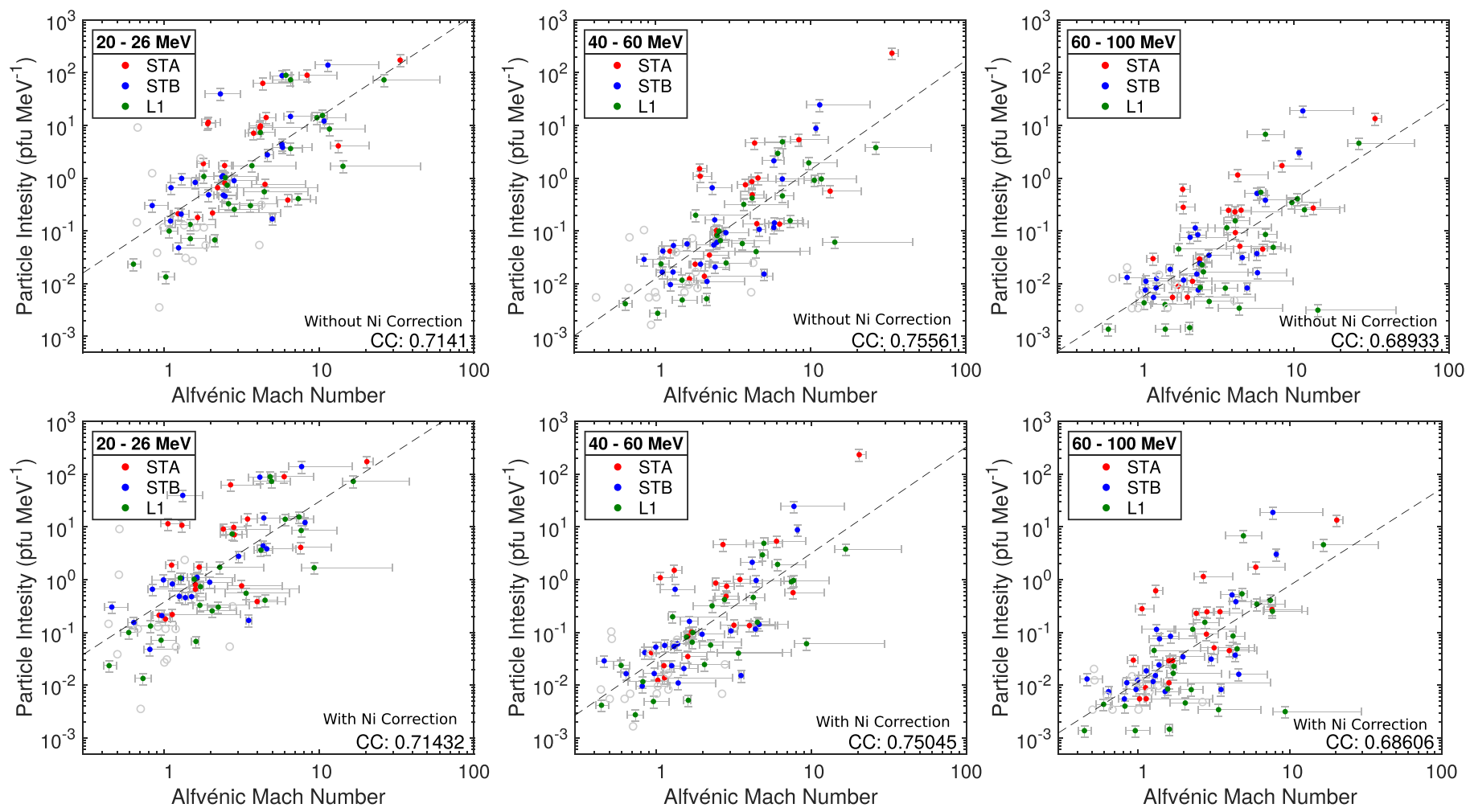

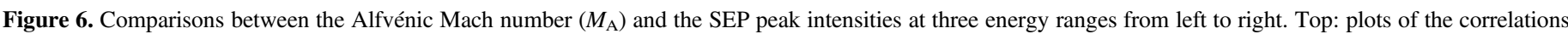

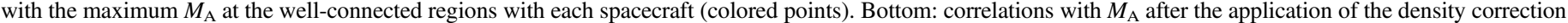
factors. The gray circles denote cases that are rejected from the analysis (see the text for details). The dashed lines are power-law fittings to the measurements.

respectively. For the density and the scattering center compression ratio the correlations are good, with CCs ranging from $\sim 53 \%$ to $\sim 62 \%$. For the magnetic compression ratio we find lower CCs, from $\sim 47 \%$ to $\sim 55 \%$. In all the cases the CCs for the compression ratios are lower than the $\mathrm{CCs}$ we find for the shock speed or the Mach numbers.

From the statistics in Figure 8 it is important to note the following: the issue encountered with the correlations is that the density compression ratio saturates fast with increasing values of the peak intensities, and the points cluster in a narrow region between $X \sim 3.5$ and 4 . This clustering results from the solutions of the R-H jump conditions. When the Mach number tends to values beyond 10 , the compression ratio tends to an asymptotic value of 4 when the polytropic index $\gamma=5 / 3$.

The relatively good correlations in both the scattering center and the density compression ratio suggest that efficient acceleration is expected to occur in regions of increased Mach number. Both compression ratios, $X$ and $r_{c}$, depend on the magnitude of $M_{\mathrm{A}}$ and the shock geometry. Therefore, our survey based on 33 SEPs detected supports the conclusions of the case studies in Rouillard et al. (2016) and Plotnikov et al. (2017). Along with the $M_{\mathrm{A}}$ number, $r_{c}$ is a critical parameter in simulations of DSA (Vainio et al. 2014; Afanasiev et al. 2018). The best CCs are found for the scattering center compression ratio at $40-60 \mathrm{MeV}(\mathrm{CC} \sim 64 \%)$, while in any energy range the $\mathrm{CCs}$ of the magnetic compression ratio are the lowest found here.

\subsection{Correlation of SEP $\mathrm{I}_{\text {peak }}$ with Shock $\theta_{B N}$}

We follow a different approach to investigate the link between $\theta_{\mathrm{Bn}}$ and Ip. Instead of using the maximum value of
$\theta_{\mathrm{BN}}$ in the well-connected region, we use the value of $\theta_{\mathrm{BN}}$ at the time when $M_{\mathrm{A}}$ is maximum or the shock becomes supercritical. The reason that drives us to switch our method is the time dependence of the $\theta_{\mathrm{BN}}$ in the well-connected regions. In most of the cases, when the shock connects for the first time with the open magnetic field lines, the geometry is quasi-perpendicular in the well-connected regions. Progressively as the shock further evolves, its geometry at the connected region becomes more oblique, and finally it switches to quasi-parallel shock.

In Figure 9 we show the correlations between Ip and the $\theta_{\mathrm{Bn}}$ value at the time of (1) maximum $M_{\mathrm{A}}$ (red points) and (2) $M_{\mathrm{A}}=M_{\mathrm{A}}(C)$ (black points). For condition 1 we find a weak anticorrelation, while for condition 2 we find a weak correlation of $\theta_{\mathrm{Bn}}$ with the Ip. The $\mathrm{CC}$ values in any of those cases are the worst found so far (see Table 2). The absolute CC values are lower than $30 \%$. From the results of Figure 9, it is inconclusive whether the shock geometry can be connected with the acceleration efficiency. A more detailed timedependent analysis is needed to further elucidate this aspect. Some predictions of the DSA theory show that highly oblique shocks have a higher acceleration efficiency compared to shocks that are nearly aligned along the magnetic field (e.g., Jokipii 1987; Giacalone 2005), while other DSA theoretical models (Zank et al. 2000; Lee 2005) and simulations (Vainio et al. 2014; Afanasiev et al. 2018) predict that protons accelerated by quasi-parallel shocks stream upstream away from the shock and generate self-excited Alfvén waves that scatter particles back to the shock for further acceleration (Vainio \& Laitinen 2007). 

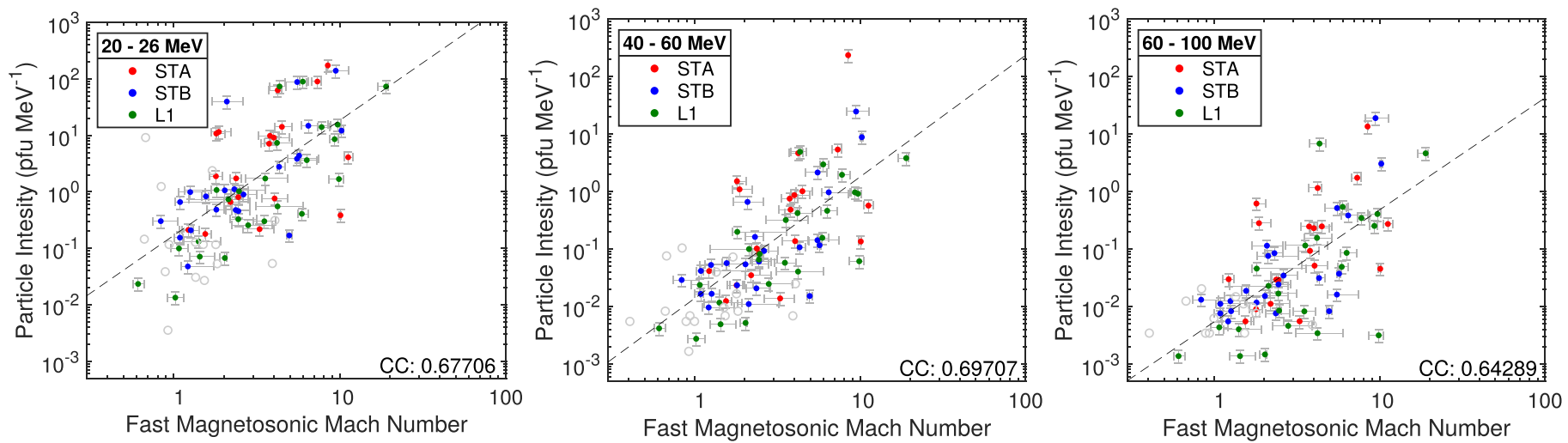

Figure 7. Comparisons between the SEP peak intensities and the fast magnetosonic Mach number $\left(M_{\mathrm{fm}}\right)$. See Figure 6 for more details.

\subsection{Relating the Shock Wave Evolution to SEP Release Times}

We further examine the relative time difference between the estimated release time of the $\mathrm{S} / \mathrm{C}$-specific SEP events and the time when the shock waves reach the well-connected field lines for each S/C. We use Tables 4 and 5 of Paassilta et al. (2018), which include the SEP release times for the events in our list. Those times were inferred from the application of either the velocity dispersion analysis (VDA) or the time-shifting analysis (TSA) to the SEPs' onset times.

We perform this timing analysis by selecting a characteristic time when the shock waves are both connected to the S/C and sufficiently strong to accelerate particles to high energy. From the temporal evolution of the shock parameters at the wellconnected field lines we register for each S/C-specific SEP event the time when the shock wave becomes supercritical $\left(M>M_{c}\right)$ (and connected to the S/C). Since the threshold for criticality depends on local plasma conditions $\beta$ and geometry $\theta_{\mathrm{BN}}$, and because for $\beta<1$ the $M_{c}$ varies from $\sim 1.53$ for parallel shocks and $\sim 2.76$ for perpendicular shocks, we choose an average value of $M_{c} \sim 2$ to infer the time when a wellconnected region at the shock front is supercritical or not.

In the top panel of Figure 10 we show the distributions of the computed time difference, $\Delta T$, between the SEP release time and the time when the shock becomes supercritical. We have rejected the cases in Paassilta et al. (2018) when the release times have an uncertainty greater than 60 minutes. From the timing distributions of the top panel, we find a maximum at -15 minutes for both VDA and TSA and a median around -11 minutes from VDA and -23 minutes from TSA, i.e., the SEP release occurs on average later than the time when the well-connected shock waves become supercritical along specific lines. This time difference must to a large extent be attributed to the time for the particles to be accelerated to high energy by the shock. The scatter of timings around the distribution maximum/mean values arises mainly from two likely sources: (1) the uncertainty in the methods used to estimate SEP release time (mean errors of $\sim 30$ minutes; see Paassilta et al. 2018), and (2) the inherent uncertainty from the selection of the critical Mach number threshold. Additionally, there are cases for which release times cannot be explained from the evolution of the shock wave parameters only.

In the bottom panel of Figure 10 we show the time difference as a function of the absolute longitudinal angular separation between the $\mathrm{S} / \mathrm{C}$ magnetic connection point at the shock and the eruptive active region, $\Delta \Phi$. We have found for all the triangulated shock waves that the heliocentric position of the eruptive active region is generally close to the direction of propagation and therefore the apex of the CME. For $\Delta \Phi<70^{\circ}$ the absolute time differences range from a few minutes up to $\sim 80$ minutes, with median values of $\sim 10-15$ minutes (excluding the three outliers). For $\Delta \Phi>70^{\circ}$ the time differences become greater than 90 minutes and the particle release is difficult to explain from the evolution of the shock wave. Those extreme cases (three for VDA and eight for TSA) with high time differences must be a product of at least two effects. There are typically large uncertainties involved for the estimation of the SEP release time when the particle flux measured in situ rises very gradually in comparison with other events that exhibit prompt and sharp onsets in particle fluxes. The other is likely due to the actual acceleration/transport mechanisms at play. The pressure waves we model far from the apex are no longer shocks but rather fast-mode waves; consequently, spacecraft at these longitudes connect magnetically to likely inefficient accelerators. We discuss these points further in the next section.

\subsection{SEP Acceleration and Release at Distant Connected S/C}

In the left panel of Figure 11 we show the relation between the maximum Alfvén Mach number $M_{\mathrm{A}}$ (black points) and maximum shock speed (red points) with respect to the separation angle $\Delta \Phi$ defined in the previous paragraph. The two horizontal lines depict the thresholds for $M_{\mathrm{A}}>1$ (dotdashed line) and $M_{\mathrm{A}}>M_{c}$ (dashed line).

An anticorrelation between $M_{\mathrm{A}} /$ shock speed and $\Delta \Phi$ is observed in the left panel of Figure 11. In addition to the anticorrelation, the range of $M_{\mathrm{A}}$ /shock speed values decreases as $\Delta \Phi$ increases. For SEPs with good magnetic connectivity, i.e., low $\Delta \Phi$ values, the magnetic field lines are connected closer to the wave apex where the shock is strongly driven. The dynamic range of possible shock parameters is controlled by the driver (flux rope) characteristics. As $\Delta \Phi$ increases, the magnetic connectivity is displaced toward the flanks of the CME event, where the pressure wave becomes detached from the driver and progressively evolves into a freely propagating wave. The observed reduction of the range of $M_{\mathrm{A}} /$ shock speed therefore corresponds to the transition from a fast-driven motion to a more freely propagating motion at the local characteristic speed (see Figure 11).

The results of Figure 11 (left) show that for $\Delta \Phi<120^{\circ}$ supercritical shocks are measured in all but eight of the 

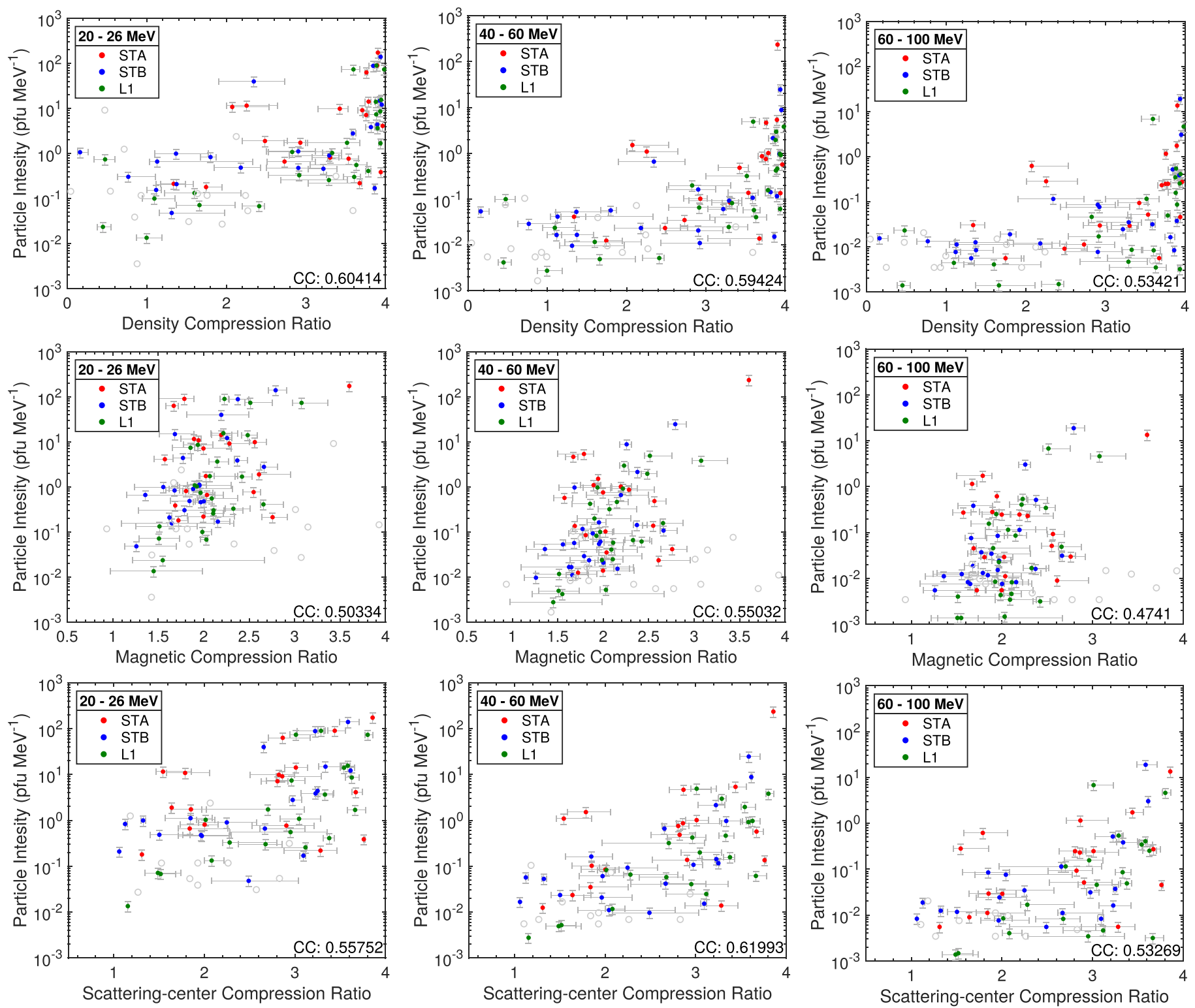

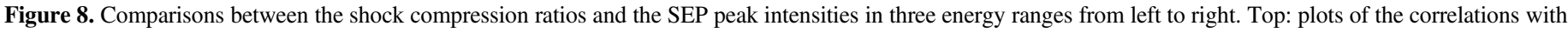

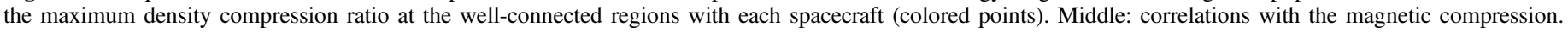
Bottom: correlations with the scattering center compression. The gray circles denote cases that are rejected from the analysis (see the text for details).

magnetic connections. For $\Delta \Phi \sim 90^{\circ}-120^{\circ}$, there are cases where a connection to a supercritical shock region is still measured; however, for higher $\Delta \Phi$ values $\left(>120^{\circ}\right)$ we find only four instances of supercritical shocks.

In the middle panel of Figure 11 we present, as a function of $\Delta \Phi$, the height where the shock becomes supercritical (red points) and where $M_{\mathrm{A}}$ maximizes (black points) along the field lines connected to spacecraft measuring SEPs. Supercriticality is reached between $\sim 1.2$ and $6 R_{\odot}$, and the shock waves reach their maximum strength (in terms of maximum $M_{\mathrm{A}}$ ) at heights well below $\sim 20 R_{\odot}$. We also examined whether there is any dependence of the observed particle intensity on the connection height where the shock becomes supercritical (Figure 11, right panel). We found a weak anticorrelation between the connection height and the Ip, which suggests that the most intense SEPs are produced in shock regions that become supercritical low in the corona.

\section{Summary and Discussion}

In this study, we carried out a systematic survey of the relationship between the properties of modeled shock waves and SEPs.

Previous studies by Kahler (2001), Kahler \& Vourlidas (2013), Richardson et al. (2015), and Papaioannou et al. (2016) have established a good correlation between the SEP peak intensities and CME speeds. The CCs between the CME parameters and the SEP characteristics usually varied from $\sim 40 \%$ to $\sim 70 \%$, and they were significantly better than the correlations with the flare parameters. Despite the good correlation between CME speeds and SEP characteristics established in previous work, single CME parameters cannot capture the complexity of shocks and how they connect to in situ detectors. In this paper we reexamined those relations in a more comprehensive manner. Additionally, we 

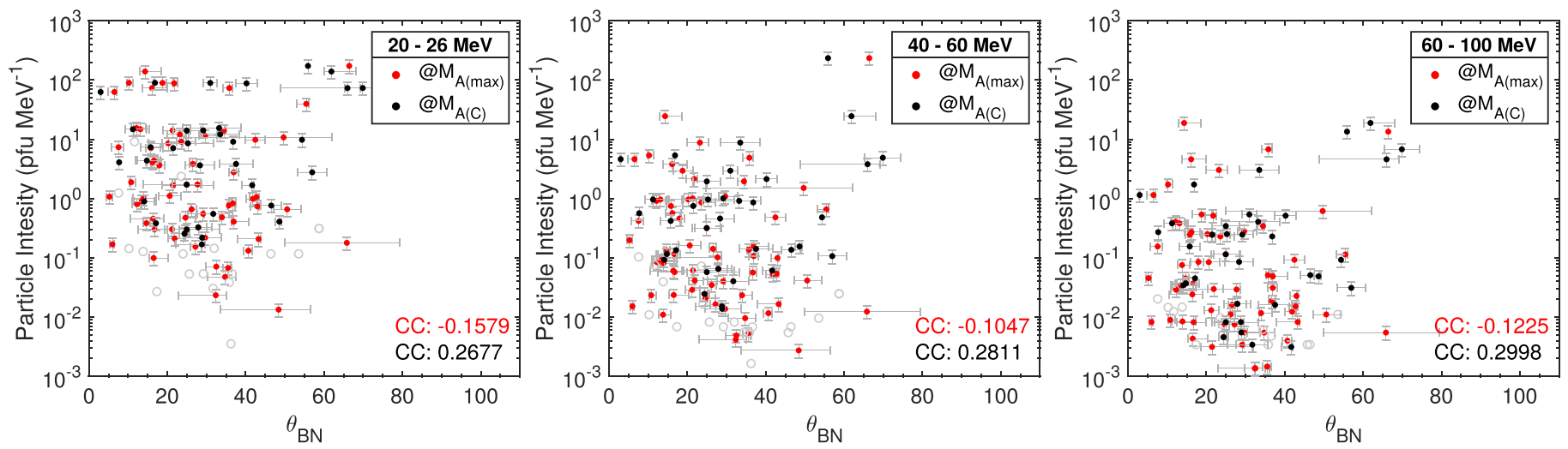

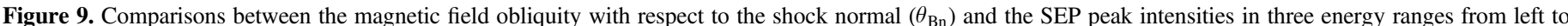
right.
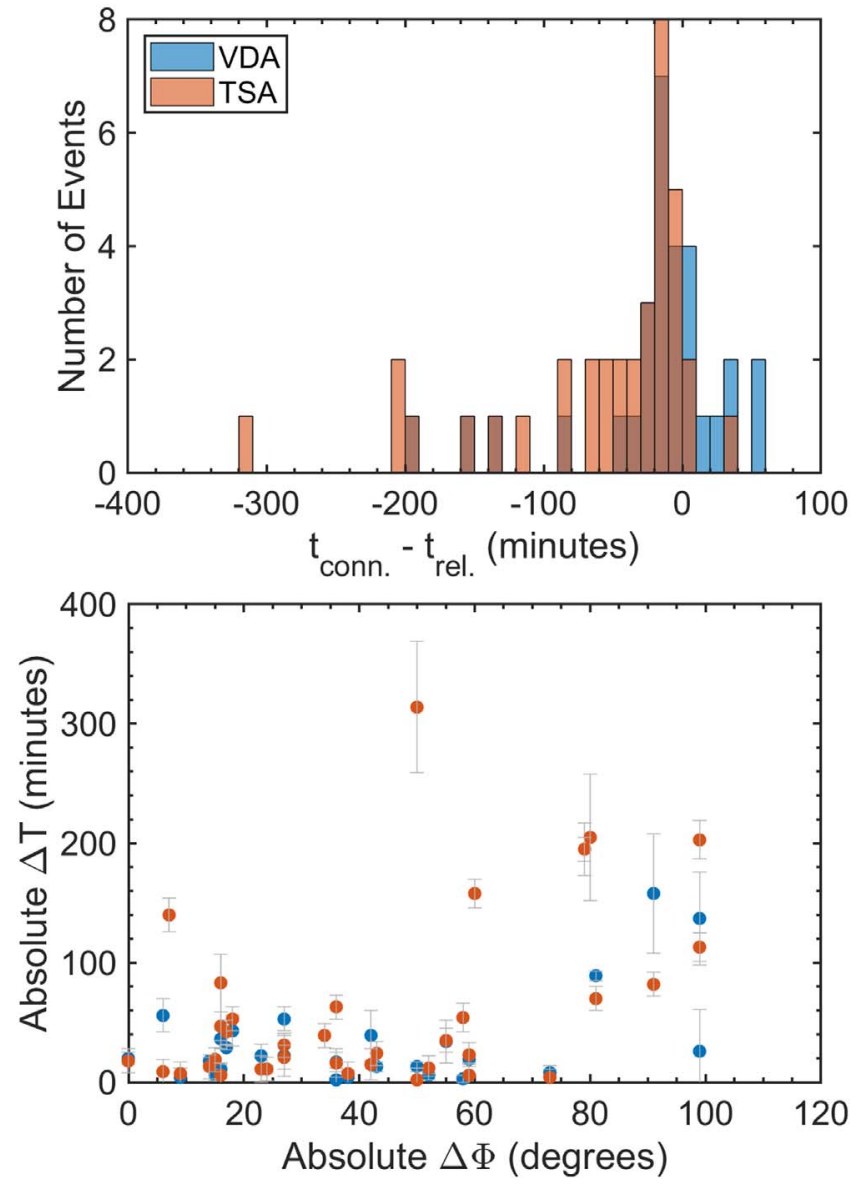

Figure 10. Top: distributions of the relative time difference, $\Delta T$, between the SEP release time from VDA and TSA and the time when the shock becomes supercritical and is connected to the $\mathrm{S} / \mathrm{C}$. Bottom: absolute $\Delta T$ using VDA (blue points) and TSA (red points) as a function of the absolute longitudinal angular separation between the $\mathrm{S} / \mathrm{C}$ well-connected region and the erupting active region, $\Delta \Phi$.

investigated correlations with other shock parameters that are relevant for particle acceleration such as the shock Mach numbers, the compression ratios, and shock geometry $\left(\theta_{\mathrm{BN}}\right)$.

First, we showed that the correlations between the SEP peak intensity and the shock (or CME) speeds improved significantly (in a statistical sense) compared with previous studies when magnetic connectivity and 3D shock properties are considered. An improvement of the correlations was also found when we compared the Ip with the shock Mach numbers. The CCs between Ip and the Alfvénic Mach number are the highest among all shock parameters considered in this study, with CCs from $69 \%$ up to $76 \%$. This result supports the conclusions drawn by Rouillard et al. (2016), namely, that efficient particle acceleration likely occurs in regions of very high Mach numbers (see also Reames 2012; Plotnikov et al. 2017). The high correlations with Alfvénic Mach number and the good correlations with the scattering center compression ratio give further support to the results of recent DSA simulations of Afanasiev et al. (2018). We have found no evidence for any significant energy dependence for $M_{\mathrm{A}}$ and shock speed to exist at the relatively moderate energies studied here below $100 \mathrm{MeV}$.

Figures 6 and 7 show that there is nevertheless some significant scatter that points to other likely contributing factors to the variability of SEP events. These may include a role of shock geometry, the nature of seed particles, and SEP transport. We have also examined the correlations with $\theta_{\mathrm{BN}}$. The resulting correlations are not statistically significant, and an apparent relation is difficult to establish from Figure 9. Recent studies have shown that for a realistic coronal structure the seed particles gain energy gradually by interacting with a rapidly varying shock geometry (e.g., Sandroos \& Vainio 2009; Kong et al. 2017). A proper evaluation of the role of shock geometry on the acceleration process requires a more refined analysis that folds in time dependency. We note that the analysis presented, while accounting for the time-varying shock properties along specific field lines, does not address the time dependence of the acceleration process itself.

In Section 3.5 we further examined the properties of the evolving shock wave with the SEP release time at the wellconnected field lines for each $\mathrm{S} / \mathrm{C}$. The results of Figure 10 illustrate the connection between the expanding shock wave and the properties of SEPs even for cases widely separated from the parent ARs (e.g., $\Delta \Phi \sim 90^{\circ}$ ). For events with very far magnetic connections (e.g., $\Delta \Phi \gg 90^{\circ}$ ) the timings suddenly worsen significantly. These timings do not support a link between the evolution of the wave and the measured particle onsets. We note, however, that in these cases difficulties arise in the estimation of the SEP release times because particle fluxes rise very gradually and can remain close to the background fluxes. 

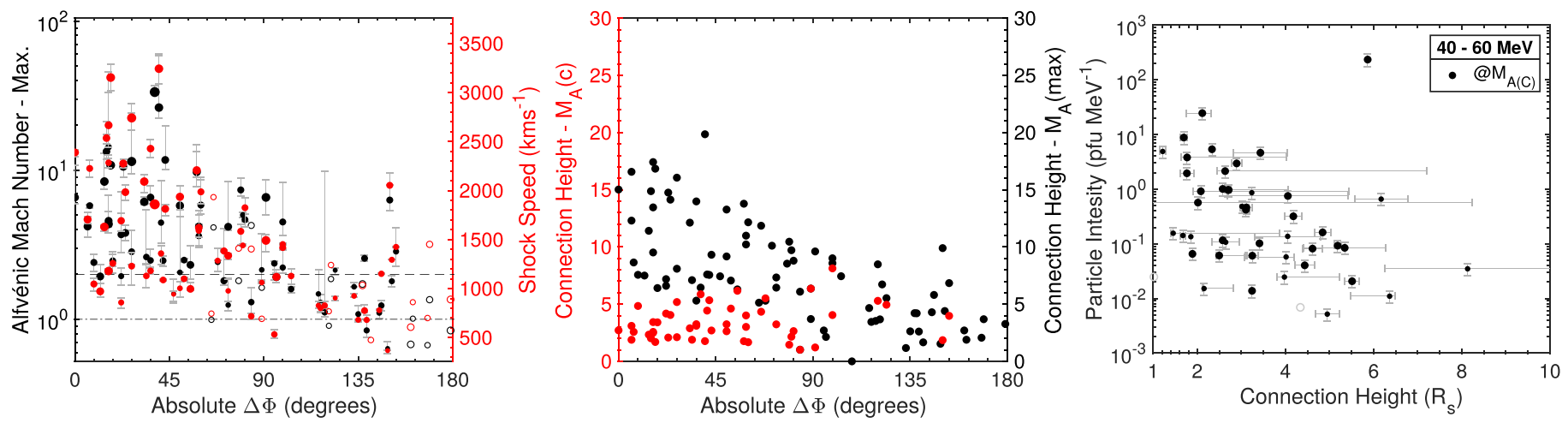

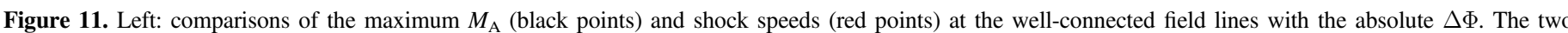

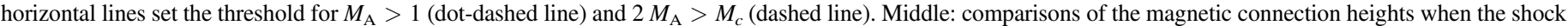

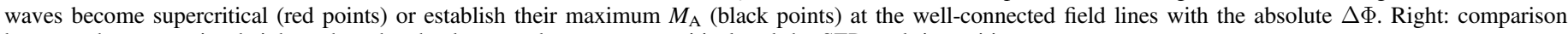
between the connection heights when the shock waves become supercritical and the SEP peak intensities.

Additionally, in Section 3.6 we examined the shock wave properties as a function of $\Delta \Phi$. The wide extent of strong shock waves highlighted in Section 3.6 must be connected to the broad longitudinal extent of SEPs. In this respect, it is worth noting that for cases where $\Delta \Phi \gg 120$ and the connected shock wave regions are not supercritical (or even no shocks, $M_{\mathrm{A}}<1$ ), they share common characteristics. Those particle onset times occur much later than the start of the eruptions, and they additionally exhibit low Ip values, just above the background. For those cases, particle transport mechanisms (including cross-field diffusion) may influence the times of particle releases. We showed also that the maximum shock strength is established at the well-connected field lines at moderate to low heights in the corona, between $\sim 1.5$ and $20 R_{\odot}$.

We have also investigated the possible influence of other pre-event CMEs. First, we checked whether other CMEs occurred in coronal images before and during every modeled shock wave. When a CME occurred within $12 \mathrm{hr}$ prior to the onset of the modeled shock wave, we flagged our analysis as potentially affected by these other CMEs. For example, previous CMEs could have a direct impact on the SEP characteristics through the provision of pre-energized seed particles. In Figure 12 we repeated the correlation analysis between the $M_{\mathrm{A}}$ and the Ip40 taking into account the presence of CMEs (highlighted as red points). From this figure it seems that there is no apparent ordering of the measurements. If previous events had a significant impact on the resulting Ip40, a significant grouping of the points should exist between the two different indices such that red points in Figure 12 would be mostly grouped in the higher Ip regime.

Despite all these progresses, this type of analysis suffers nevertheless from a number of different difficulties that are related to the distances between the corona and the points of in situ measurements. We provided estimates of the uncertainties associated with the determination of shock parameters and of the connectivity. For instance, we worked toward improving the accuracy of the simulated coronal parameters by comparing the simulated upstream magnetic field and density with in situ measurements. In the framework of the two new solar missions, PSP and Solar Orbiter (SolO) will certainly shed new light on the structural and kinematic properties of the shock waves close to the Sun, the accuracy of the 3D reconstruction techniques and their further improvement, and our understanding of the coronal structuring and the validity of the MHD simulations,

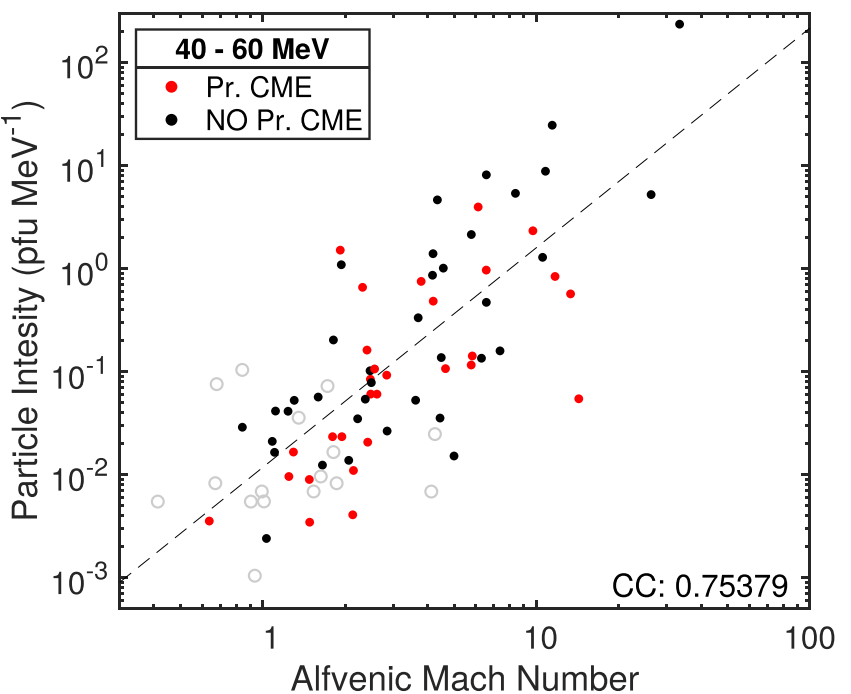

Figure 12. Comparison between the Alfvénic Mach number and the SEP peak intensities at 40-60 MeV. Red points denote cases where a previous CME was present before the SEP event, and black points denote cases without previous $\mathrm{CME}$. The gray circles denote cases that are rejected from the analysis (see the text for details).

especially for the density distributions in different coronal regions.

\section{Conclusion}

Our results and conclusions can be summarized as follows:

1. Simple correlations between the SEP peak intensities and the CME (projected in the sky plane) or deprojected shock speed at the apex give CCs of $\approx 54 \%$. When magnetic connectivity is accounted for using the techniques presented here, the Ip-shock speed correlations are significantly improved, with CCs of $\approx 65 \%$.

2. The highest correlations are obtained between the shock Mach numbers and SEP peak intensities. These correlations are significantly higher (CCs of $\approx 72 \%$ ) than with other shock parameters. We here confirm predictions of DSA that efficient acceleration of SEPs should naturally occur at shock regions where $M_{\mathrm{A}}$ is high.

3. Complementarily to the value of the correlation index, we also found that the peak intensity in SEPs has a quadratic dependence on the shock Alfvénic Mach number: 
$I_{p} \propto M_{\mathrm{A}}^{k_{1}}$, with $k_{1} \approx 2$. This dependence is consistent with the expectation of efficient DSA, with particle acceleration time being proportional to its gyro-time (Bohm regime), i.e., $\tau_{\text {acc }} \propto E /\left(e B_{1}\right)$, where $E$ is the particle energy and $B_{1}$ is the local magnetic field strength. Combined with the assumption that accelerated particles propagate from the shock front to 1 au along the flux tubes that exhibit an expansion factor $\propto 1 / B_{1}$, the total energy of accelerated particles is then expected to scale as $V_{\mathrm{sh}}^{2} / B_{1}^{2} \propto M_{\mathrm{A}}^{2}$, when measured at $1 \mathrm{au}$.

4. The compression ratios versus the peak intensities show significant correlations $(\approx 56 \%)$, albeit smaller than those obtained for the shock speed. We have not found any correlation between $\theta_{\mathrm{BN}}$ and particle peak intensities with CCs less than $30 \%$ and low $p$-values.

5. We have found no clear energy dependence on the correlations for any of the shock parameters. Overall, the highest CCs were found between shock parameters for particle intensities in the energy range of $40-60 \mathrm{MeV}$.

6 . The solar particle release times tend to occur $\sim 15-20$ minutes after the shock waves become supercritical and have connected magnetically with the SEP detector.

7. For moderate to low longitudinal separation angles $(\Delta \Phi<120)$ between the source longitude of the CME (typically the flare) and the shock location connected magnetically to the detectors measuring SEPs, the shock reaches supercriticality. The acceleration and release of SEPs could therefore be supported by the shock wave expansion to those broad regions. For $\Delta \Phi>120$, we found that overall the shock wave expansion cannot account for SEPs measured in situ, since the magnetic connection is established with subcritical regions.

8. Shock waves become supercritical at a median distance of $3 R_{\odot}$, and they reach their maximum strength until $20 R_{\odot}$.

Ignoring time-dependent effects associated with the acceleration process, we conclude that high particle fluxes are observed along magnetic field lines connecting with high Mach numbers, shock speeds, shock compression ratios, and low oblique $\theta_{\mathrm{BN}}$, in decreasing order of importance.

This study highlights the importance of improving our knowledge of $3 \mathrm{D}$ plasma coronal parameters where the strongest particle acceleration occurs and the necessary improvement in our understanding of magnetic field connectivity. The new catalog of 3D shocks established here allows us to investigate further the timing, longitudinal spread, and composition of SEPs. The still ambiguous relative roles of flares and shock waves as particle accelerators will also be addressable in more detail in future studies. $P S P$ and $S o l O$ will perform their first measurements close to the Sun over the next few years, and our 3D reconstruction and modeling work will be directly folded in the type of analysis presented here.

The IRAP team acknowledges support from the French space agency (Centre National des Etudes Spatiales; CNES; https://cnes.fr/fr) that funds activity in plasma physics data center (Centre de Données de la Physique des Plasmas; CDPP; http://cdpp.eu/) and the Multi Experiment Data \& Operation Center (MEDOC; https://idoc.ias.u-psud.fr/ MEDOC), and the space weather team in Toulouse (SolarTerrestrial Observations and Modeling Service; STORMS; https://stormsweb.irap.omp.eu/). This includes funding for the data mining tools AMDA (http://amda.cdpp.eu/) and
CLWEB (clweb.cesr.fr/) and the propagation tool (http:// propagationtool.cdpp.eu). A.K. and Y.W. acknowledge financial support from the ANR project SLOW_SOURCE (ANR18-ERC1-0006-01), COROSHOCK (ANR-17-CE31-0006-01), and FP7 HELCATS project https://www.helcats-fp7.eu/ under FP7 EU contract No. 606692. The work at the University of Turku was conducted in the framework of the Finnish Centre of Excellence in Research of Sustainable Space, funded by the Academy of Finland (grant 312357). R.V. and A.A. also acknowledge the financial support of the Academy of Finland of the project 309939. We thank the STEREO: IMPACT, SECCHI, S/WAVES; SOHO: LASCO, ERNE; Wind/ WAVES; and SDO/AIA teams and Predictive Science Inc. for providing the data used in this study. The STEREO SECCHI data are produced by a consortium of RAL (UK), NRL (USA), LMSAL (USA), GSFC (USA), MPS (Germany), CSL (Belgium), IOTA (France), and IAS (France). SOHO is a mission of international cooperation between ESA and NASA. The SDO/AIA data are provided by the Joint Science Operations Center (JSOC) Science Data Processing (SDP). The present work has also benefited from discussions held at the International Space Science Institute (ISSI, Bern, Switzerland) within the frame of the international team "High EneRgy sOlar partICle events analysis (HEROIC)" led by Dr. A. Papaioannou.

\section{ORCID iDs}

Angelos Vourlidas (i) https://orcid.org/0000-0002-8164-5948

\section{References}

Afanasiev, A., Vainio, R., Rouillard, A. P., et al. 2018, A\&A, 614, A4 Bemporad, A., \& Mancuso, S. 2011, ApJL, 739, L64

Brueckner, G. E., Howard, R. A., Koomen, M. J., et al. 1995, SoPh, 162, 357 Cane, H. V., \& Lario, D. 2006, Coronal Mass Ejections (Berlin: Springer), 45 Cane, H. V., Reames, D. V., \& von Rosenvinge, T. T. 1988, JGR, 93, 9555 Cane, H. V., von Rosenvinge, T. T., Cohen, C. M. S., \& Mewaldt, R. A. 2003, GeoRL, 30, 8017

Colaninno, R. C., \& Vourlidas, A. 2009, ApJ, 698, 852

de Patoul, J., Foullon, C., \& Riley, P. 2015, AGUFM, SH11A-2381

Desai, M. I., Mason, G. M., Mazur, J. E., \& Dwyer, J. R. 2006, SSRv, 124, 261

Dresing, N., Gómez-Herrero, R., Klassen, A., et al. 2012, SoPh, 281, 281

Giacalone, J. 2005, ApJL, 628, L37

Giacalone, J., Jokipii, J. R., \& Mazur, J. E. 2000, ApJL, 532, L75

Howard, R. A., Moses, J. D., Vourlidas, A., et al. 2008, SSRv, 136, 67

Jokipii, J. R. 1987, ApJ, 313, 842

Kahler, S., Burkepile, J., \& Reames, D. 1999, ICRC, 6, 248

Kahler, S. W. 2001, JGR, 106, 20947

Kahler, S. W., \& Vourlidas, A. 2005, JGRA, 110, A12S01

Kahler, S. W., \& Vourlidas, A. 2013, ApJ, 769, 143

Kong, X., Guo, F., Giacalone, J., Li, H., \& Chen, Y. 2017, ApJ, 851, 38

Kouloumvakos, A., Patsourakos, S., Hillaris, A., et al. 2014, SoPh, 289, 2123

Kouloumvakos, A., Patsourakos, S., Nindos, A., et al. 2016, ApJ, 821, 31

Kozarev, K. A., \& Schwadron, N. A. 2016, ApJ, 831, 120

Kwon, R.-Y., \& Vourlidas, A. 2017, ApJ, 836, 246

Kwon, R.-Y., \& Vourlidas, A. 2018, JSWSC, 8, A08

Kwon, R.-Y., Zhang, J., \& Olmedo, O. 2014, ApJ, 794, 148

Lario, D., Kwon, R.-Y., Richardson, I. G., et al. 2017, ApJ, 838, 51

Lario, D., Kwon, R.-Y., Vourlidas, A., et al. 2016, ApJ, 819, 72

Lario, D., Raouafi, N. E., Kwon, R.-Y., et al. 2014, ApJ, 797, 8

Lee, M. A. 2005, ApJS, 158, 38

Lemen, J. R., Title, A. M., Akin, D. J., et al. 2012, SoPh, 275, 17

Lionello, R., Linker, J. A., \& Mikić, Z. 2009, ApJ, 690, 902

Livadiotis, G. 2015, ApJ, 809, 111

Ontiveros, V., \& Vourlidas, A. 2009, ApJ, 693, 267

Paassilta, M., Papaioannou, A., Dresing, N., et al. 2018, SoPh, 293, 70

Papaioannou, A., Anastasiadis, A., Kouloumvakos, A., et al. 2018, SoPh, 293, 100

Papaioannou, A., Sandberg, I., Anastasiadis, A., et al. 2016, JSWSC, 6, A42 
Patsourakos, S., Georgoulis, M. K., Vourlidas, A., et al. 2016, ApJ, 817, 14

Plotnikov, I., Rouillard, A. P., \& Share, G. H. 2017, A\&A, 608, A43

Reames, D. V. 1999, SSRv, 90, 413

Reames, D. V. 2012, ApJ, 757, 93

Reames, D. V. 2013, SSRv, 175, 53

Reames, D. V. 2018, SSRv, 214, 61

Richardson, I. G., von Rosenvinge, T. T., Cane, H. V., et al. 2014, SoPh, 289, 3059

Richardson, I. G., von Rosenvinge, T. T., \& Cane, H. V. 2015, SoPh, 290, 1741

Riley, P., Lionello, R., Linker, J. A., et al. 2011, SoPh, 274, 361

Rouillard, A., \& Lockwood, M. 2004, AnGeo, 22, 4381

Rouillard, A. P., Lockwood, M., \& Finch, I. 2007, JGRA, 112, A05103

Rouillard, A. P., Plotnikov, I., Pinto, R. F., et al. 2016, ApJ, 833, 45

Rouillard, A. P., Sheeley, N. R., Tylka, A., et al. 2012, ApJ, 752, 44

Rušin, V., Druckmüller, M., Aniol, P., et al. 2010, A\&A, 513, A45

Sandroos, A., \& Vainio, R. 2007, ApJL, 662, L127

Sandroos, A., \& Vainio, R. 2009, A\&A, 507, L21

Thernisien, A., Vourlidas, A., \& Howard, R. A. 2009, SoPh, 256, 111
Torsti, J., Valtonen, E., Lumme, M., et al. 1995, SoPh, 162, 505

Tylka, A. J., Cohen, C. M. S., Dietrich, W. F., et al. 2005, ApJ, 625, 474

Tylka, A. J., \& Lee, M. A. 2006, ApJ, 646, 1319

Vainio, R., \& Laitinen, T. 2007, ApJ, 658, 622

Vainio, R., Pönni, A., Battarbee, M., et al. 2014, JSWSC, 4, A08

Vainio, R., Raukunen, O., Tylka, A. J., Dietrich, W. F., \& Afanasiev, A. 2017, A\&A, 604, A47

von Rosenvinge, T. T., Reames, D. V., Baker, R., et al. 2008, SSRv, 136, 391

von Rosenvinge, T. T., Richardson, I. G., Reames, D. V., et al. 2009, SoPh, 256, 443

Vourlidas, A., Balmaceda, L. A., Stenborg, G., \& Dal Lago, A. 2017, ApJ, 838,141

Wang, T., Reginald, N. L., Davila, J. M., St., Cyr, O. C., \& Thompson, W. T. 2017, SoPh, 292, 97

Wuelser, J.-P., Lemen, J. R., Tarbell, T. D., et al. 2004, Proc. SPIE, 5171, 111

Wurz, P., Balogh, A., Coffey, V., et al. 2007, ISSIR, 7, 117

Zank, G. P., Rice, W. K. M., \& Wu, C. C. 2000, JGR, 105, 25079

Zhang, M., Jokipii, J. R., \& McKibben, R. B. 2003, ApJ, 595, 493 